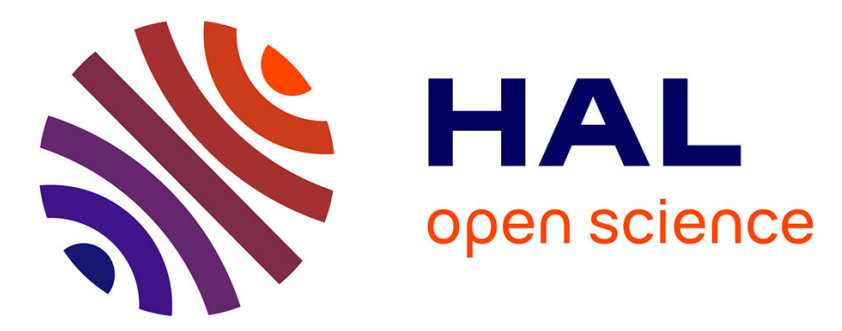

\title{
Heterogeneity of neuroblastoma cell identity defined by transcriptional circuitries
}

\author{
Valentina Boeva, Caroline Louis-Brennetot, Agathe Peltier, Simon Durand, \\ Cecile Pierre-Eugene, Virginie Raynal, Heather Etchevers, Sophie Thomas, \\ Alban Lermine, Estelle Daudigeos-Dubus, et al.
}

\section{To cite this version:}

Valentina Boeva, Caroline Louis-Brennetot, Agathe Peltier, Simon Durand, Cecile Pierre-Eugene, et al.. Heterogeneity of neuroblastoma cell identity defined by transcriptional circuitries. Nature Genetics, 2017, 49 (9), pp.1408-1413. 10.1038/ng.3921 . hal-01741718

\author{
HAL Id: hal-01741718 \\ https://hal.science/hal-01741718
}

Submitted on 26 Mar 2018

HAL is a multi-disciplinary open access archive for the deposit and dissemination of scientific research documents, whether they are published or not. The documents may come from teaching and research institutions in France or abroad, or from public or private research centers.
L'archive ouverte pluridisciplinaire HAL, est destinée au dépôt et à la diffusion de documents scientifiques de niveau recherche, publiés ou non, émanant des établissements d'enseignement et de recherche français ou étrangers, des laboratoires publics ou privés. 
4 Authors: Valentina Boeva ${ }^{1,2, *}$, Caroline Louis-Brennetot ${ }^{3}$, Agathe Peltier ${ }^{3}$, Simon Durand ${ }^{3}$, 5 Cécile Pierre-Eugène ${ }^{3}$, Virginie Raynal ${ }^{3,4}$, Heather Etchevers ${ }^{5}$, Sophie Thomas ${ }^{6}$, Alban

6 Lermine $^{1}$, Estelle Daudigeos-Dubus ${ }^{7}$, Birgit Geoerger ${ }^{7}$, Martin F. Orth $^{8}$, Thomas G. P.

7 Grünewald $^{8}$, Elise Diaz ${ }^{9,10}$, Bertrand Ducos ${ }^{9,10,11}$, Didier Surdez ${ }^{3}$, Angel M. Carcaboso ${ }^{12}$, Irina 8 Medvedeva $^{2}$, Thomas Deller ${ }^{13}$, Valérie Combaret ${ }^{14}$, Eve Lapouble ${ }^{15}$, Gaelle Pierron ${ }^{15}$, Sandrine 9 Grossetête-Lalami ${ }^{3}$, Sylvain Baulande ${ }^{4}$, Gudrun Schleiermacher ${ }^{3,16,17}$, Emmanuel Barillot ${ }^{1}$, 10 Hermann Rohrer $^{13}$, Olivier Delattre ${ }^{3}$, and Isabelle Janoueix-Lerosey ${ }^{3, *}$

\section{Affiliations}

$15{ }^{1}$ Institut Curie, PSL Research University, Inserm U900, Mines-ParisTech, F-75005, Paris, 16 France.

17 Institut Cochin, Inserm U1016, CNRS UMR 8104, University Paris Descartes UMR-S1016, F18 75014, Paris, France.

$19{ }^{3}$ Institut Curie, PSL Research University, Inserm U830, F-75005, Paris, France.

$20{ }^{4}$ Institut Curie Genomics of Excellence (ICGex) Platform, Institut Curie Research Center, F21 75005, Paris, France.

$22{ }^{5}$ Aix Marseille Univ, Inserm, GMGF, UMR S910, F-13005, Marseille, France.

$23{ }^{6}$ Inserm U1163, Laboratory of Embryology and Genetics of Congenital Malformations, Paris 24 Descartes - Sorbonne Paris Cité University, Imagine Institute, F-75015 Paris, France.

$25{ }^{7}$ Vectorology and Anticancer Therapies, UMR 8203, CNRS, Univ. Paris-Sud, Gustave Roussy, 26 Université Paris-Saclay, F-94805, Villejuif, France.

$27{ }^{8}$ Max-Eder Research Group for Pediatric Sarcoma Biology, Institute of Pathology, LMU 28 Munich, Thalkirchner Str. 36, 80337 Munich, Germany.

$29{ }^{9}$ High Throughput qPCR facility, IBENS, PSL Research University, 46 rue d'Ulm, F-75005, 
Paris, France.

${ }^{10}$ LPS-ENS, UPMC, Université Denis Diderot, CNRS UMR 8550, PSL 24 rue Lhomond, F75005, Paris, France.

$33{ }^{11}$ Laser Microdissection Facility, CIRB Collège de France, Place Marcellin Berthellot, F-75005, 34 Paris, France.

$35 \quad{ }^{12}$ Institut de Recerca Sant Joan de Deu, 08950 Barcelona, Spain.

$36{ }^{13}$ Institute for Clinical Neuroanatomy, Goethe University, D-60590, Frankfurt/M, Germany.

$37 \quad{ }^{14}$ Centre Léon Bérard, Laboratoire de Recherche Translationnelle, Lyon, France.

$38 \quad{ }^{15}$ Institut Curie, Unité de Génétique Somatique, F-75005, Paris, France.

$39 \quad{ }^{16}$ Laboratory RTOP (Recherche Translationnelle en Oncologie Pédiatrique), laboratoire

40 "Gilles Thomas", Institut Curie, F-75005, Paris, France.

$41 \quad{ }^{17}$ Department of translational research, Institut Curie, F-75005, Paris, France.

44 Neuroblastoma is a tumor of the peripheral sympathetic nervous system ${ }^{1}$, derived from 45 multipotent neural crest cells (NCCs). To define Core Regulatory Circuitries (CRCs) controlling 46 the gene expression program of neuroblastoma, we established and analyzed the neuroblastoma 47 super-enhancer landscape. We discovered three types of identity in neuroblastoma cell lines: a 48 sympathetic noradrenergic identity defined by a CRC module including the PHOX2B, HAND2 49 and GATA3 transcription factors (TFs); an NCC-like identity, driven by a CRC module 50 containing AP-1 family TFs; a mixed type further deconvoluted at the single cell level.

51 Treatment of the mixed type with chemotherapeutic agents resulted in enrichment of NCC-like 52 cells. The noradrenergic module was validated by ChIP-seq. Functional studies demonstrated 53 dependency of neuroblastoma with noradrenergic identity on PHOX2B, evocative of lineage 54 addiction. Most neuroblastoma primary tumors express TFs from the noradrenergic and NCC55 like modules. Our data demonstrate a novel aspect of tumor heterogeneity relevant for 56 neuroblastoma treatment strategies.

58 Keywords: neuroblastoma, neural crest cells, ChIP-seq, super-enhancers, core regulatory 59 circuitries, transcription factors, cell proliferation 
Nearly one in six patients who die of a childhood cancer had a neuroblastoma, a tumor of

62 the peripheral sympathetic nervous system ${ }^{1}$. Several genes including $M Y C N^{2}, A L K^{3-6}$ and $63 T E R T^{7,8}$ have been shown to act as major drivers of neuroblastoma oncogenesis. In this work, we 64 have determined the core transcriptional regulatory circuitries $(\mathrm{CRCs})^{9}$ that govern the gene 65 expression program of neuroblastoma. CRCs, which can be defined by super-enhancer (SE) 66 mapping of H3K27 acetylation mark (H3K27ac) and further sequence motif analysis, provide 67 integrative information about cell identity ${ }^{9,10}$.

68 We examined a panel of twenty-five neuroblastoma cell lines (Table S1) and two primary human neural crest cell (hNCC) lines ${ }^{11}$. SEs were defined by the ROSE algorithm ${ }^{12}$ modified to account for copy number changes. Principal Component Analysis (PCA), based on scores of SEs identified in at least two neuroblastoma cell lines or in both hNCC lines $(n=5975)$ revealed two distinct groups (Figure 1a): group I with 18 neuroblastoma cell lines and group II comprising the GIMEN, SH-EP and GICAN neuroblastoma cell lines. Group II closely resembled the hNCC lines in this analysis. Four neuroblastoma cell lines occupied an intermediate position between groups I and II. These included the phenotypically heterogeneous SK-N-SH cell line whereas its sub-clones, SH-SY5Y and SH-EP, were included in groups I and II, respectively. This result is consistent with SH-SY5Y cells displaying neurite-like processes and expressing noradrenergic biosynthetic enzymes TH and DBH ("N" phenotype), and SH-EP cells exhibiting a substrateadherent "S" phenotype without expression of $\mathrm{TH}$ and $\mathrm{DBH}^{13}$. We also profiled the SE landscape of six patient-derived xenografts (PDXs), five of them with MYCN amplification (Table S2). All PDXs clustered with group I when included in the PCA (Figure 1a).

SEs were then sorted according to the median H3K27ac signal for each group (Figure 1b and 1c, respectively; Table S3). In group I, the strongest SEs comprise a set of transcription 84 factor (TF) loci including HAND2, PHOX2A/PHOX2B and GATA2/GATA3 and the ALK oncogene locus (Figure 1b, 1d, 1e, Figures S1 and S2). These findings are consistent with previous SE data on a few neuroblastoma cell lines ${ }^{14,15}$. PHOX2B, HAND2, and GATA3 are

87 known to participate in a complex $\mathrm{TF}$ network controlling normal sympathetic neuron 88 specification and differentiation ${ }^{16,17}$. Recurrent SEs in these TFs therefore appear to be a 89 hallmark of sympathetic cell identity. Most SEs of group II overlapped with SEs of hNCC lines 90 (Figure 1c), consistent with the results of the PCA analysis. 
MYCN amplification, and 3 PDX (Figure S3). No groups linked to the MYCN or ALK status were revealed in the PCA. Furthermore, supervised analysis of SE scores did not indicate SEs associated with MYCN amplification or ALK mutations (Tables S4 and S5). Cell lines with a PHOX2B mutation were observed in group I (SH-SY5Y), group II (SH-EP) or in the intermediate group (SK-N-SH).

To detect driver TFs for groups I and II, we used i-cisTarget to find DNA sequence motifs enriched in the SEs with the highest score. For group I, this analysis identified a TAATYYAATTA binding motif common to several homeobox proteins, including PHOX2B and PHOX2A (Figure S4). During sympathetic nervous system development PHOX2B regulates PHOX2A expression ${ }^{18}$. Both TFs are highly expressed in most neuroblastoma cell lines and primary tumors (Figure S5). PHOX2B was undetectable at the transcript and protein level solely in the group II cell lines as well as in the hNCC lines, but was expressed in all other neuroblastoma cell lines (Figure 1f; Figure S6). There was a corresponding lack of SE at PHOX2B and no TH and DBH transcription in GIMEN, SH-EP and GICAN (Figure S7). IcisTarget analysis showed enrichment in AP-1 motif in group II and in the hNCC lines (Figures S8 and S9). AP-1 is a heterodimer composed of FOS and JUN family members both of which are expressed in immature $\mathrm{hNCC}^{11}$. These results suggest that $\mathrm{PHOX} 2 \mathrm{~B}$ participates in the activity of neuroblastoma group I SEs while the AP-1 complex TFs influence the SE landscape of group II.

A CRC calling algorithm ${ }^{9,19}$ identified PHOX2B as group I-specific CRC TF and FOSL1, FOSL2 and JUN for group II (Figure S10), consistent with our i-cisTarget results. We therefore searched for TFs predicted to be in a CRC either with PHOX2B or a FOS/JUN family member (Figure 1g). Cell lines showing an intermediate position in the PCA had a CRC that included several TFs of both PHOX2B-associated and FOS/JUN-associated sets. Most of TFs of the latter set are expressed in neural crest cells and/or mesenchymal neural crest derivatives. The CRCs of the six PDXs were highly similar to those of the group I cell lines (Figure 1g). As we documented that SE strength linearly correlated with gene expression (Figure S11), we used the latter to further define fully connected TF modules. This analysis revealed two main TF modules distinguishing groups I and II (Figure 1h). PHOX2B, GATA3 and HAND2 were present in module 1 whereas module 2 included FOSL1 and FOSL2. These modules were anti-correlated at the gene expression level. Western blot analysis confirmed the co-expression of several TFs in 
group I or group II (Figure S12). Furthermore, single cell analysis showed that the SK-N-AS and SK-N-SH cell lines are heterogeneous and comprise cells expressing TFs of either module 1 or module 2 within the same population (Figure 1i, Figure S13, Table S6).

Taken together, these data demonstrate a novel type of heterogeneity in neuroblastoma cell lines and suggest that individual cells assume either a sympathetic noradrenergic identity, characterized by a CRC module including PHOX2B, HAND2 and GATA3, and subsequent expression of the enzymes $\mathrm{TH}$ and/or DBH; or an NCC-like identity, characterized by expression of a distinct module including FOS and JUN family members but lacking PHOX2B and noradrenergic marker expression. Both types of identity are observed in several heterogeneous cell lines. All cell lines with MYCN amplification except one (CHP-212) had a noradrenergic identity whereas cell lines without MYCN amplification displayed any of the three identities (Figure 1g).

To explore whether the different identity classes seen in neuroblastoma cell lines are also characteristic for neuroblastoma primary tumors, expression data from a large set of primary tumors ( $\mathrm{n}=498$; dataset GSE49711) ${ }^{20}$ were studied. Correlations between expression of the TFs of each module identified in cell line CRCs were calculated for these primary tumors. We found strong positive correlations between PHOX2B, HAND2, and GATA3 of module 1 as well as between the TFs of the NCC-like module (Figure 2a). We also observed anti-correlations between PHOX2B, HAND2, and GATA3 on the one hand, and TFs of the NCC-like module on the other hand. These results therefore confirm the data obtained with cell lines and further define a PHOX2B/HAND2/GATA3 noradrenergic CRC module in primary neuroblastomas. Next, we used the average expression of the two modules to explore primary tumor identity. All but two tumors showed high expression of the noradrenergic module (Figure 2b). A continuum was observed between low to high values of the NCC-like module, suggesting heterogeneity of cell identity in primary tumors. The remaining two cases with low noradrenergic and high NCClike module expression may correspond to rare cases with full NCC-like identity, as described for group II cell lines. Similar to the cell lines (Figure S14), lower expression of the NCC-like module was observed in the majority of MYCN-amplified tumors (two-sided Wilcoxon signedrank test p-value $1.01 \times 10^{-10}$ ). A role for MYCN in downregulation of genes from this module is consistent with its promotion of peripheral neuron differentiation from multipotent avian $\mathrm{NCC}^{21}$. 
15410 diagnosis/relapse sample pairs ${ }^{22}$. Different patterns were observed between the two disease 155 stages (Figure 2c). To address a possible link between heterogeneity of cell identity and treatment response, we investigated the effect of chemotherapy on the NCC-like SH-EP and 157 noradrenergic SH-SY5Y cell lines. SH-EP cells were more resistant to the three agents used 158 (Figure S15). Treatment of the parental SK-N-SH cell line with doxorubicin or cisplatin resulted 159 in the respective decreased or increased expression of module 1 and 2 (Figure 2d). Enrichment of cells with an NCC-like identity thus correlates with better drug resistance. However, we cannot exclude that treatment may also induce transdifferentiation from noradrenergic to NCC-like identity. The observation that tumors at relapse are not systematically enriched in NCC-like cells supports the concept of plasticity in the reversion of cell identity. This may rely on a switch from 164 adrenergic to NCC-like identity under chemotherapy and from NCC-like to noradrenergic after 165 treatment. Altogether, these data underline the importance of targeting both types of cells during treatment.

Strong correlations between PHOX2B, HAND2 and GATA3 expression were observed 168 both in cell lines and tumors. Phox $2 b$ directly binds Hand 2 protein ${ }^{23}$, and Phox $2 b$, Hand 2 and 169 Gata3 cross-regulate during sympathetic nervous system development ${ }^{16}$. We therefore performed 170 ChIP-seq analysis for these TFs in the CLB-GA neuroblastoma cell line and identified binding 171 motifs for PHOX2B, HAND2 (zinc finger TF) and GATA3 (bHLH leucine zipper TF) (Figure 3a). Binding regions for all three TFs corresponded to the H3K27ac peaks in the PHOX2B, GATA3, HAND2 and ALK SEs and also in the MYCN SE (Figure $3 \mathrm{~b}$ and 3c, Figure S16). These results therefore confirm the biological existence of the noradrenergic module, showing that PHOX2B, HAND2 and GATA3 are SE-regulated and bind to the SEs of each other (Figure 3d). We next investigated the occupancy by these TFs of 4,336 SE regions predicted in at least two neuroblastoma cell lines. SE regions were ranked according to average SE score and intersection with TF binding sites was evaluated. Over $90 \%$ of the strong and recurrent neuroblastoma SEs were co-occupied by PHOX2B, HAND2 and GATA3 (Figure 3e). Additionally, positional 180 binding analysis showed that HAND2, PHOX2B and GATA3 bind the same 400 bp-long 181 regions within active regulatory regions (Figure 3f). Altogether, our results demonstrate that 182 PHOX2B, HAND2 and GATA3 are master TFs defining the SE landscape of neuroblastoma cell 183 lines with a noradrenergic identity.

184 It has been demonstrated that cancer dependencies can be found among SE-marked 
185

186

187

188

189

190

191

192

193

194

195

196

197

198

199

200

201

202

203

204

205

206

207

208

209

210

211

212

213

214

genes $^{19,24}$. Although missense and frameshift $P H O X 2 B$ mutations predispose to neuroblastoma ${ }^{25,26}$, its role in sporadic neuroblastoma remains poorly understood. Phox $2 \mathrm{~b}$ knockout mice completely lack autonomic structures ${ }^{17}$ whereas conditional knock-out leads to decreased neuroblast proliferation ${ }^{27}$. An effect of PHOX2B knockdown on neuroblastoma cell proliferation has been previously suggested ${ }^{28}$. To further document the consequence of PHOX2B knockdown on neuroblastoma growth, we generated a doxycycline-inducible anti-PHOX2B short-hairpin RNA (shRNA) expression system in noradrenergic CLB-GA and SH-SY5Y cells. Inducible decrease of PHOX2B protein (Figure $4 \mathrm{a}, 4 \mathrm{c}$ ) resulted in significant inhibition of neuroblastoma cell growth (Figure 4b, 4d, Figure S17). Decreased expression of PHOX2B in CLB-GA cells also impaired tumor growth in vivo (Figure 4e and 4f, Figure S18). We then evaluated whether PHOX2B decrease was sufficient to change the noradrenergic identity of the CLB-GA and SH-SY5Y cell lines to an NCC-like identity. However, data obtained by RNA-seq and RT-q-PCR suggested that the residual level of PHOX2B was sufficient to maintain a noradrenergic identity (Figure S19). This observation is consistent with the noradrenergic identity of the CLB-PE cell line in which PHOX2B expression is low but detected at the protein and RNA levels (Figures $1 \mathrm{f}$ and S6).

We observed a reduction of proliferation upon HAND2 and GATA3 knockdown in several cell lines, consistently with previous data on GATA3 knockdown ${ }^{15}$ (Figure S20). These results are in line with Hand 2 and Gata3 controlling sympathetic neuroblast proliferation ${ }^{16}$. Neuroblastoma cells of noradrenergic identity therefore appear to be addicted to these key lineage TFs as well as to PHOX2 ${ }^{29}$.

In conclusion, our work provides fundamental insights into the transcriptomic and epigenomic landscape of neuroblastoma. Distinct $\mathrm{TF}$ networks predicate different tumor identities, corresponding to sympathetic noradrenergic or NCC-like identity. Most primary tumors comprise cells of both identities, revealing a novel aspect of tumor heterogeneity. Neuroblastoma treatment should benefit from specifically targeting both identities. 
a

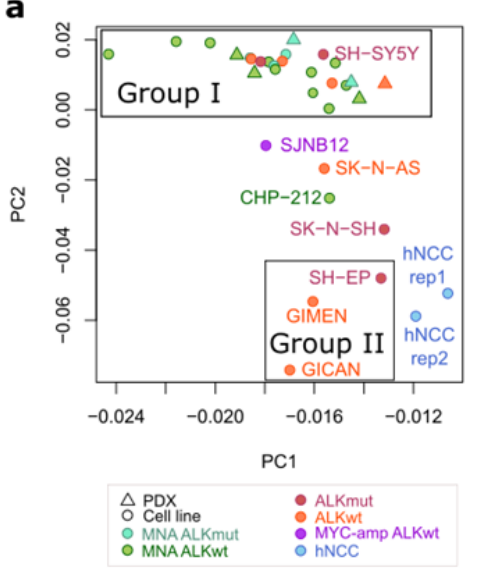

b

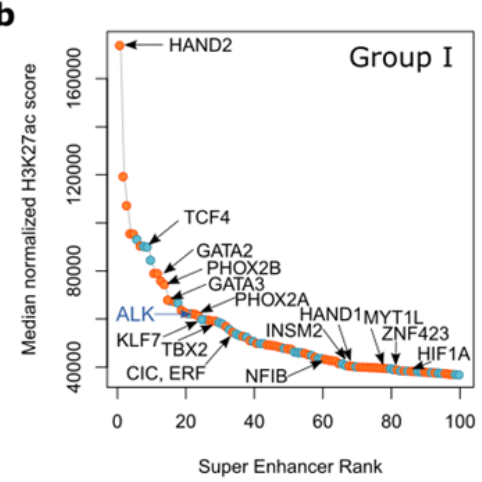

C

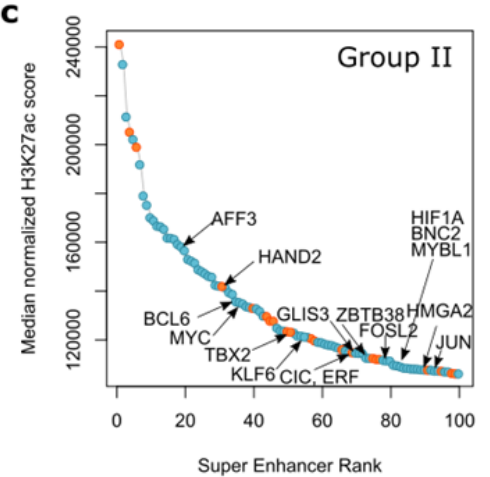

a
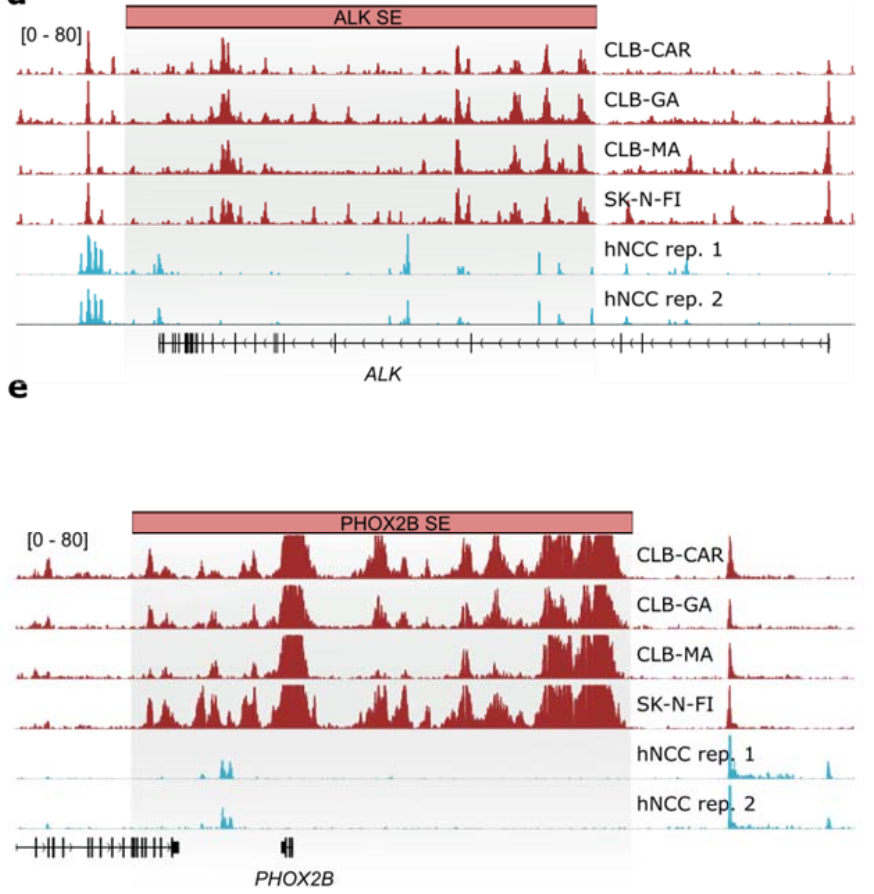

- No overlap with hNCC SEs
- Overlaps with hNCC SEs

f

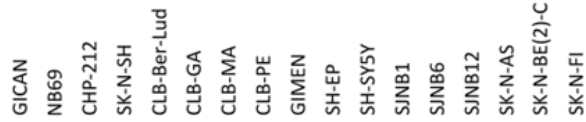
РнОх2в - - - - - - - - - -

\section{g}
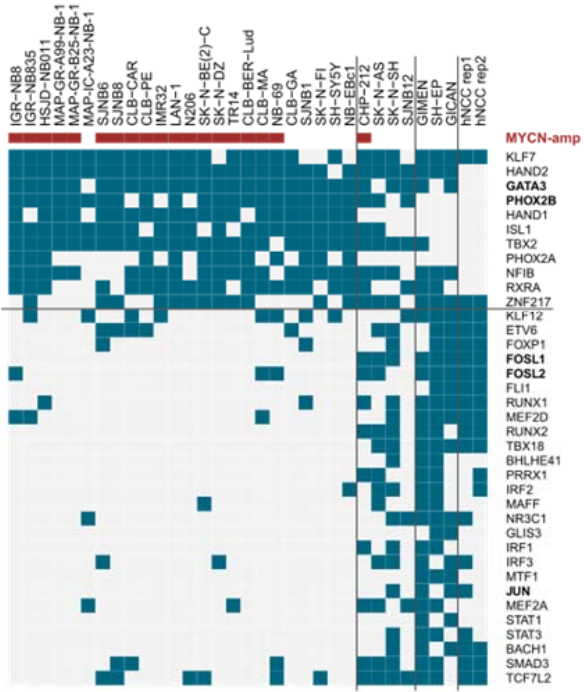

- Present in predicted CRCs absent in predicted CRCs h

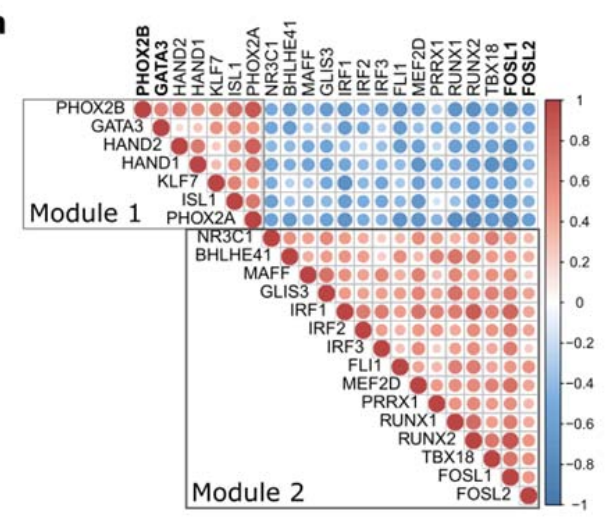

i

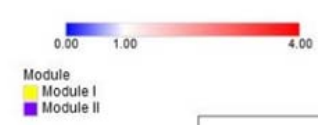

- Moovie II

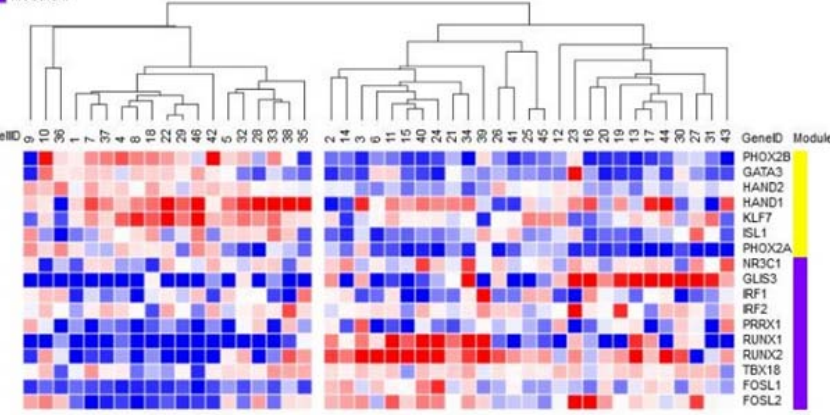


216 Figure 1. SE landscape reveals various CRCs and identities in neuroblastoma cell lines. a,

217 Principal Component Analysis (PCA) based on neuroblastoma and hNCC SE log scores. MNA:

218 MYCN amplification. b, c, Ranked plot for the 100 SEs with the highest median H3K27ac score 219 in neuroblastoma cell line groups I and II, respectively. TFs are indicated in black with arrows. 220 d, e, Tracks for ChIP-seq profiles for H3K27ac binding at $A L K$ and PHOX2B SEs, respectively. 221 f, Western blot analysis of PHOX2B and vinculin as a loading control in a panel of 222 neuroblastoma cell lines. SK-N-SH cells correspond to batch 1. g, TFs predicted to participate in 223 a CRC with PHOX2B (upper part) or with a FOS/JUN family member (lower part) in 224 neuroblastoma cell lines. TFs whose binding motifs are enriched in SEs of group I and II are 225 shown in bold. h, Pearson correlation matrix for the expression values of 22 TFs identified in 226 CRCs of cell lines shows strong positive correlations within module 1 and module 2; correlation 227 is calculated for RNA-seq data in neuroblastoma cell lines and PDX $(n=31)$. $\mathbf{i}$, Single cell 228 analysis reveals heterogeneity of cell identity in the SK-N-AS cell line. Expression of TF of 229 modules 1 and 2 was evaluated by RT-q-PCR and data were normalized to the SK-N-AS cell 230 population overall. 
a

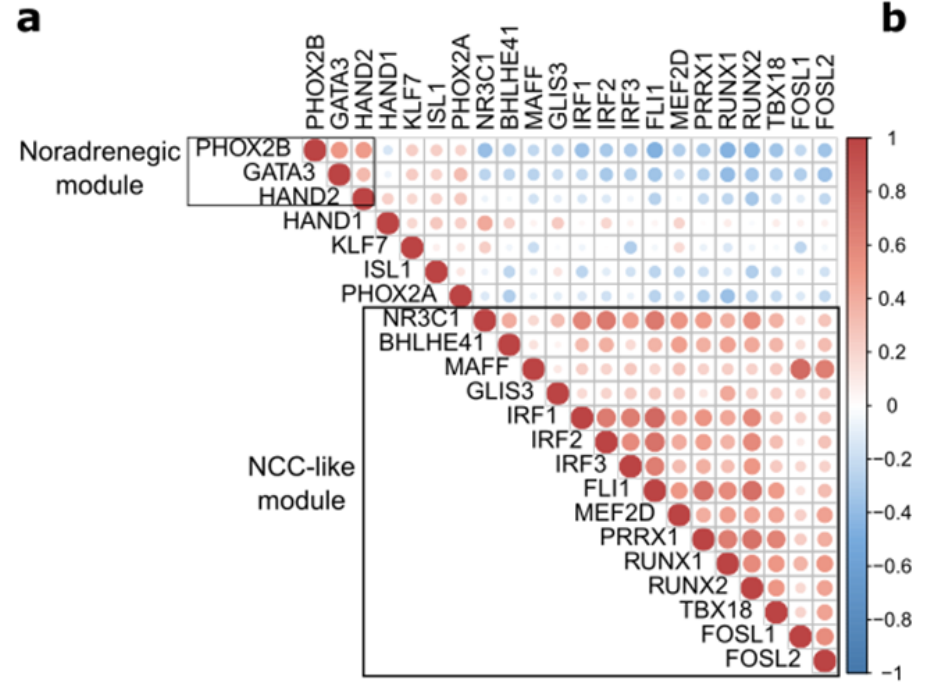

C

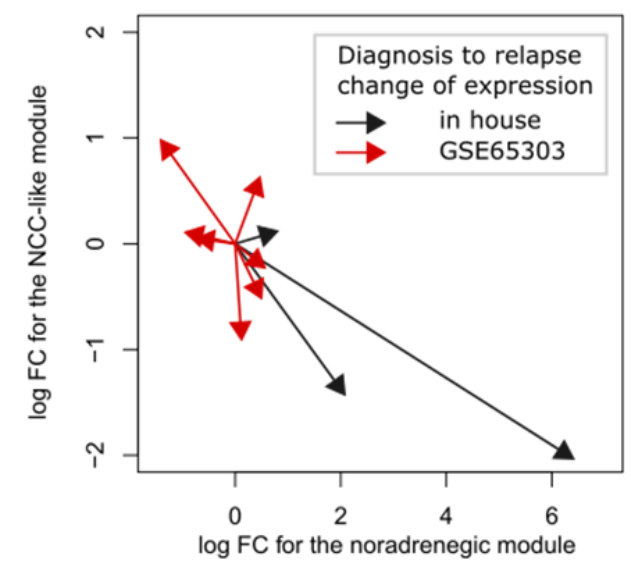

b

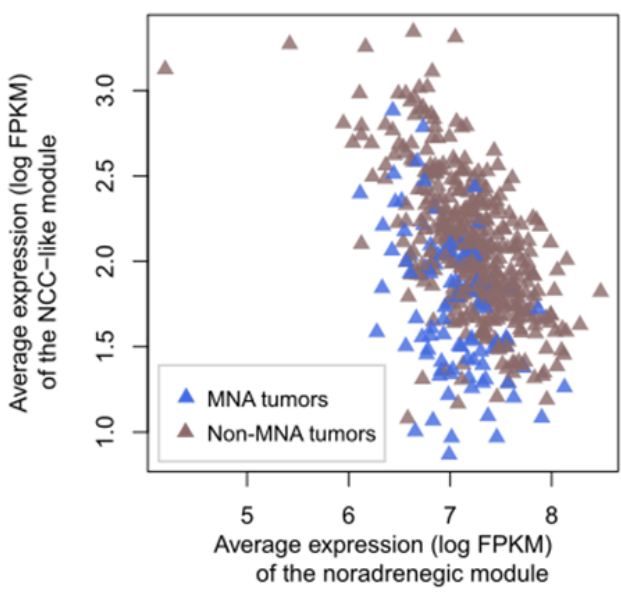

d

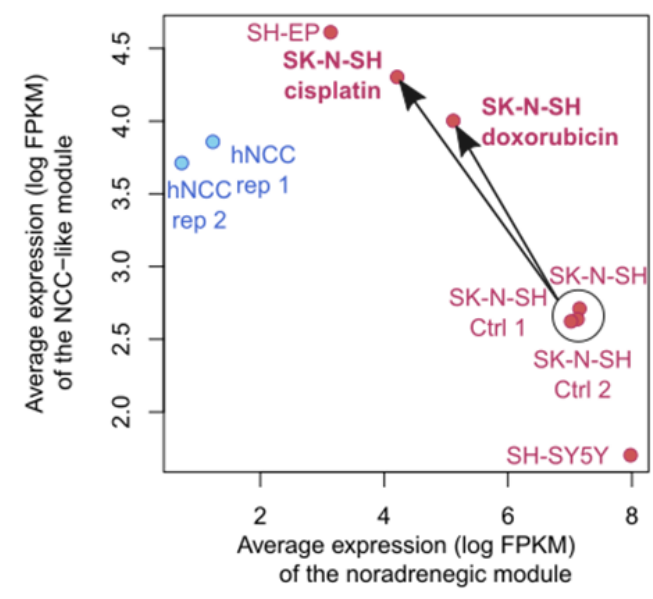

Figure 2. Different identity of neuroblastoma primary tumors and impact of chemotherapy on cell identity. a, Pearson correlation matrix for the 22 TFs identified in CRCs of cell lines in a set of 498 neuroblastoma primary tumors. b, Mean expressions of the noradrenergic and NCC-

241 like modules negatively correlate in the whole set of tumors (Pearson $\mathrm{R}=-0.49$, one-sided 242 permutation test $\mathrm{p}$-value $<10^{-10}$ ) and define a continuum between full noradrenergic and NCC243 like cases. Blue: tumors with $M Y C N$ amplification. c, Identity of tumor pairs at diagnosis and 244 relapse revealed by expression profiling. The series includes 7 pairs from the GSE65303 245 dataset $^{22}$ (red) and 3 in-house pairs (black). d, Treatment of SK-N-SH cells with chemotherapy 246 favors cells with an NCC-like identity. Cells used in this experiment (batch 2) were more 247 noradrenergic compared to the ones used in the ChIP-seq experiment (batch 1). 

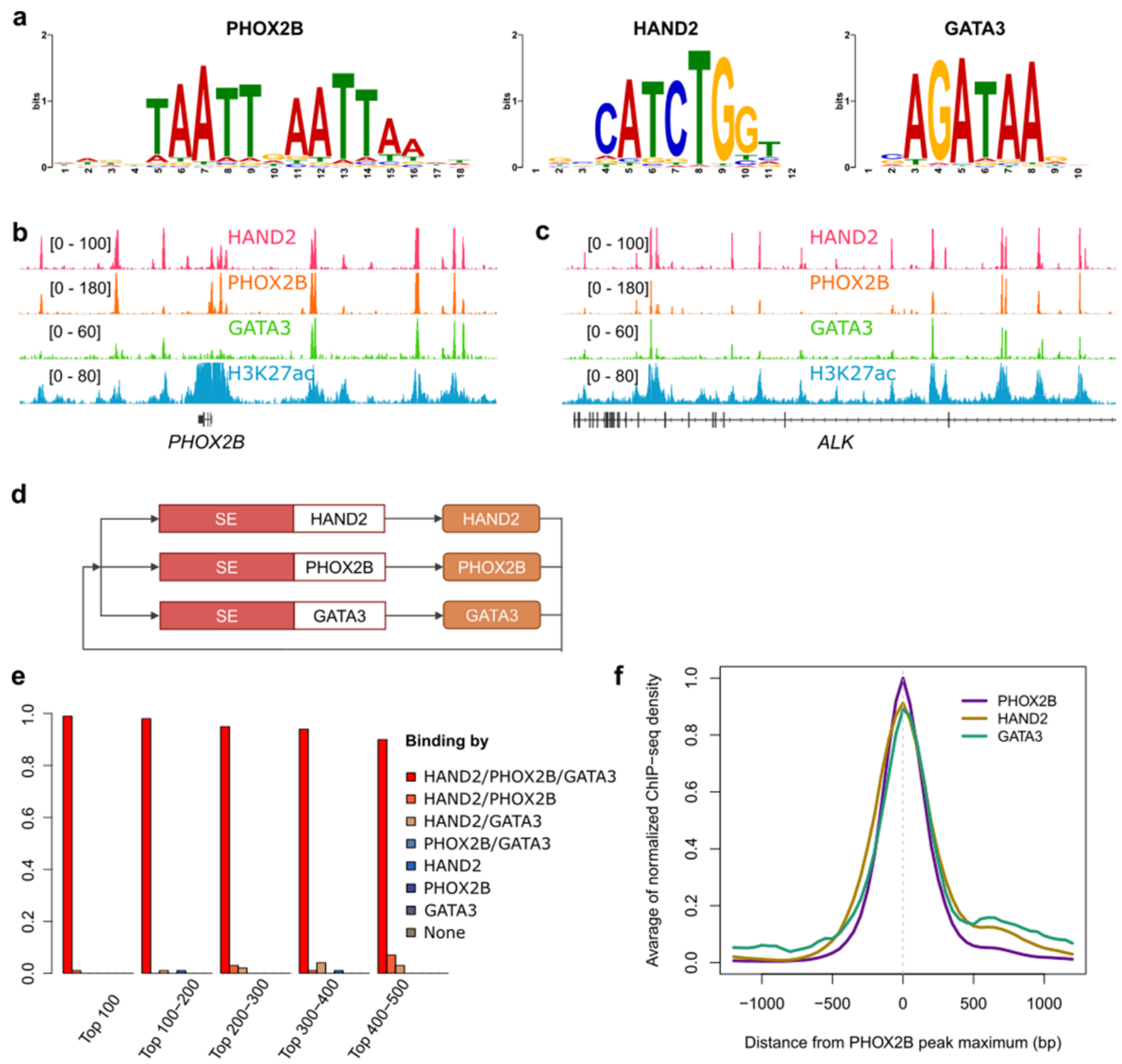
Figure 3. PHOX2B, HAND2 and GATA3 are master transcription factors defining the SE landscape of noradrenergic neuroblastoma. a, De novo identification of PHOX2B, HAND2 and GATA3 TF binding motifs. b and c, Tracks for ChIP-seq profiles for PHOX2B, HAND2, 252 GATA3 and H3K27ac binding at PHOX2B and ALK SEs, respectively. d, CRC of activating TFs 253 that define a noradrenergic module. e, Neuroblastoma SEs defined by H3K27ac peaks are 254 simultaneously occupied by PHOX2B, HAND2 and GATA3. f, HAND2, PHOX2B and GATA3 255 bind closely located regions within neuroblastoma SEs (summary of densities of 2,078 binding 256 sites corresponding to 500 top neuroblastoma SEs). 
a

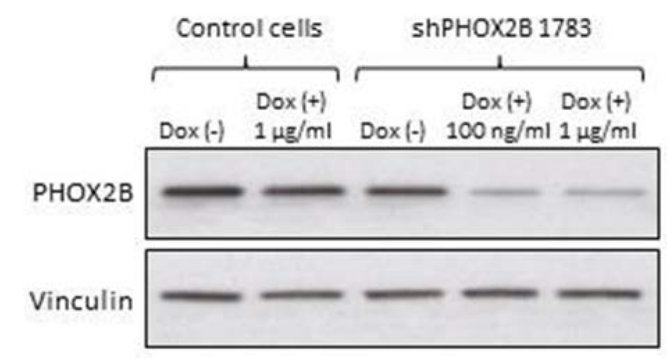

C

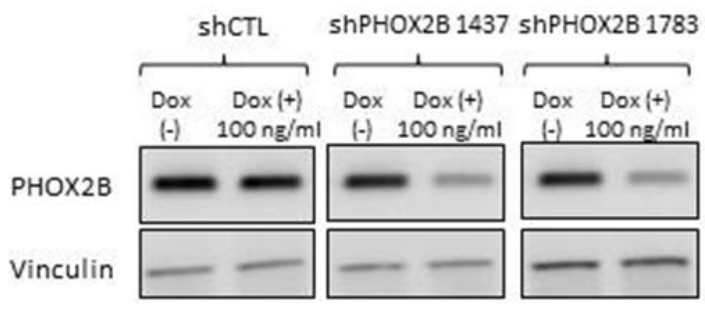

b

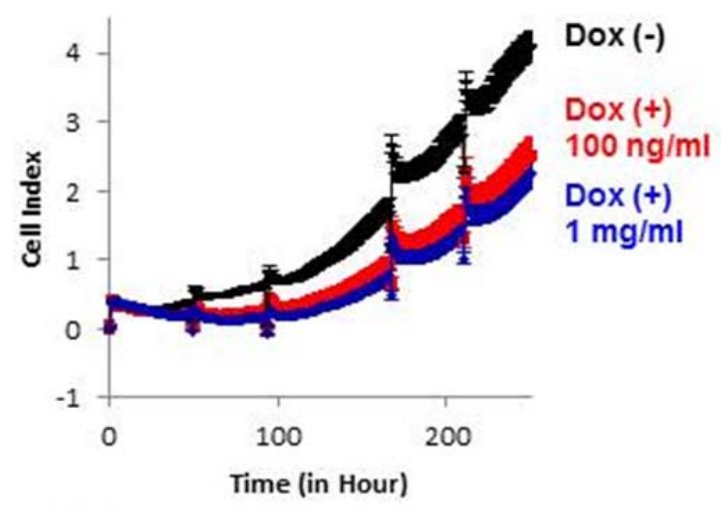

d

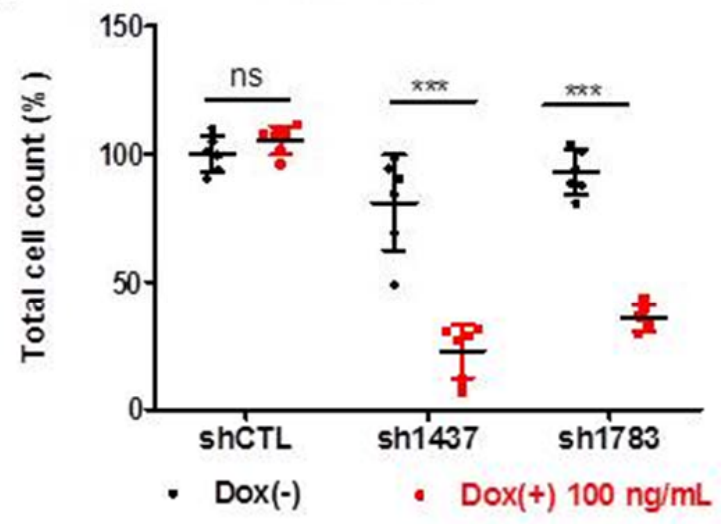

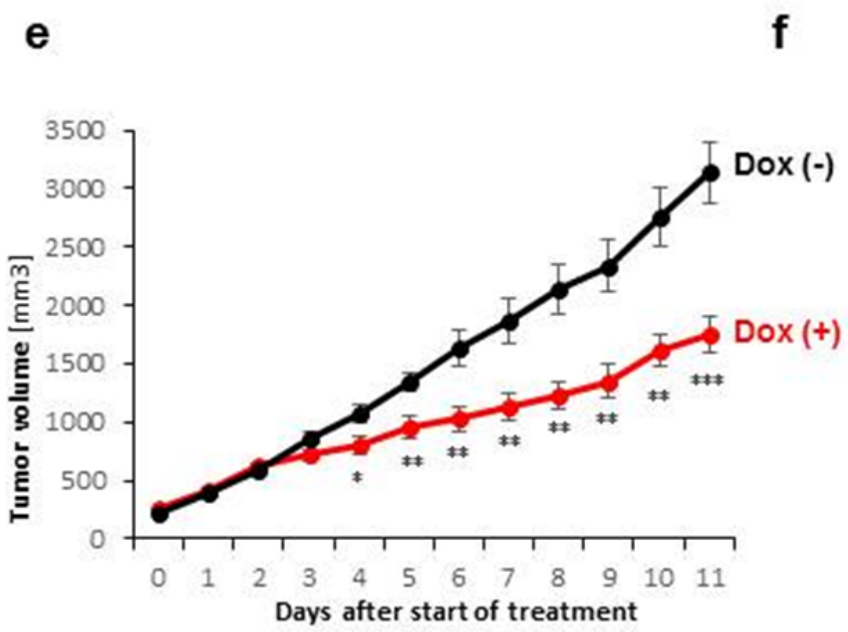

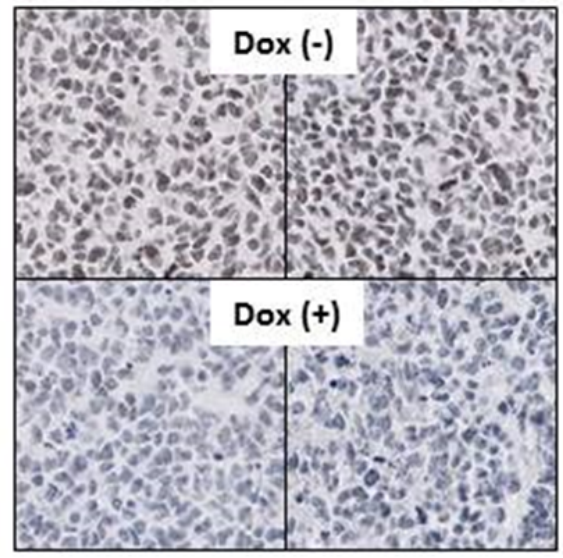

260 Figure 4. PHOX2B is critical for the growth of noradrenergic neuroblastoma cells. a, 261 PHOX2B knockdown following doxycycline treatment was confirmed at $72 \mathrm{~h}$ by immunoblot 262 (loading control: vinculin) in CLB-GA neuroblastoma cells infected with a shRNA targeting 263 PHOX2B vector. $\mathbf{b}, \mathrm{xCELLigence}{ }^{\mathrm{TM}}$ proliferation kinetics of infected CLB-GA cells in absence 
264 or presence of doxycycline at $100 \mathrm{ng} / \mathrm{ml}$ or $1 \mu \mathrm{g} / \mathrm{ml}$. Data shown are the mean \pm s.d. of results 265 obtained in the different conditions ( $\mathrm{n}=5$ technical replicates). c, PHOX2B immunoblot of SH266 SY5Y neuroblastoma cells infected with 2 different shRNA vectors targeting PHOX2B, at $72 \mathrm{~h}$. 267 d, Cell counts for the SH-SY5Y cell line infected with sh1437 or sh1783 vectors targeting 268 PHOX2B or with the control shCTL vector. $10^{5}$ cells were plated in 24 -well plates at day 0 in the 269 absence or presence of doxycycline at $100 \mathrm{ng} / \mathrm{ml}$. The number of living cells was counted at day 2708 (Mean \pm s.d.; $n=6$ replicates). e, Growth curves for subcutaneously xenografted sh1783 271 transduced CLB-GA cells. When tumors reached a volume of around $170 \mathrm{~mm} 3$, doxycycline and 272 sucrose $($ Dox + ) or sucrose alone (Dox - ) was added to the drinking water (Mean \pm s.e.m.; $\mathrm{n}=8$ 273 mice per group). P values were determined via two-tailed unpaired Welch's t-test (***: $274 \mathrm{p}<0.001$ ). f, PHOX2B immunohistochemistry (brown) combined with Hematoxylin staining in 275 two xenografts treated with doxycycline and two control xenografts.

276

277

278

279

280

281

282

283

284

285

286

287 
289 1. Matthay, K. K. et al. Neuroblastoma. Nat. Rev. Dis. Primer 2, 16078 (2016).

290 2. Brodeur, G. M., Seeger, R. C., Schwab, M., Varmus, H. E. \& Bishop, J. M. Amplification 291 of N-myc in untreated human neuroblastomas correlates with advanced disease stage. Science 292 224, 1121-4 (1984).

293 3. Mosse, Y. P. et al. Identification of ALK as a major familial neuroblastoma 294 predisposition gene. Nature 455, 930-5 (2008).

295 4. Janoueix-Lerosey, I. et al. Somatic and germline activating mutations of the ALK kinase 296 receptor in neuroblastoma. Nature 455, 967-70 (2008).

297 5. George, R. E. et al. Activating mutations in ALK provide a therapeutic target in 298 neuroblastoma. Nature 455, 975-8 (2008).

299 6. Chen, Y. et al. Oncogenic mutations of ALK kinase in neuroblastoma. Nature 455, 971-4 300 (2008).

301 7. Peifer, M. et al. Telomerase activation by genomic rearrangements in high-risk 302 neuroblastoma. Nature 526, 700-704 (2015).

303 8. Valentijn, L. J. et al. TERT rearrangements are frequent in neuroblastoma and identify 304 aggressive tumors. Nat. Genet. 47, 1411-1414 (2015).

3059 9. Saint-André, V. et al. Models of human core transcriptional regulatory circuitries. 306 Genome Res. 26, 385-396 (2016).

307 10. Hnisz, D. et al. Super-enhancers in the control of cell identity and disease. Cell 155, 934308947 (2013).

309 11. Thomas, S. et al. Human neural crest cells display molecular and phenotypic hallmarks of 310 stem cells. Hum Mol Genet 17, 3411-25 (2008).

311 12. Whyte, W. A. et al. Master transcription factors and mediator establish super-enhancers 312 at key cell identity genes. Cell 153, 307-319 (2013).

313 13. Ross, R. A., Spengler, B. A. \& Biedler, J. L. Coordinate morphological and biochemical 314 interconversion of human neuroblastoma cells. J. Natl. Cancer Inst. 71, 741-747 (1983).

315 14. Chipumuro, E. et al. CDK7 Inhibition Suppresses Super-Enhancer-Linked Oncogenic 316 Transcription in MYCN-Driven Cancer. Cell 159, 1126-1139 (2014).

317 15. Oldridge, D. A. et al. Genetic predisposition to neuroblastoma mediated by a LMO1 318 super-enhancer polymorphism. Nature 528, 418-421 (2015). 
319 16. Rohrer, H. Transcriptional control of differentiation and neurogenesis in autonomic 320 ganglia. Eur J Neurosci 34, 1563-73 (2011).

321 17. Pattyn, A., Morin, X., Cremer, H., Goridis, C. \& Brunet, J. F. The homeobox gene 322 Phox $2 \mathrm{~b}$ is essential for the development of autonomic neural crest derivatives. Nature 399, $366-$ 32370 (1999).

324 18. Flora, A. et al. Sp proteins and Phox $2 b$ regulate the expression of the human Phox $2 \mathrm{a}$ 325 gene. J. Neurosci. 21, 7037-7045 (2001).

326 19. Lin, C. Y. et al. Active medulloblastoma enhancers reveal subgroup-specific cellular 327 origins. Nature 530, 57-62 (2016).

328 20. Zhang, W. et al. Comparison of RNA-seq and microarray-based models for clinical 329 endpoint prediction. Genome Biol. 16, (2015).

330 21. Wakamatsu, Y., Watanabe, Y., Nakamura, H. \& Kondoh, H. Regulation of the neural 331 crest cell fate by N-myc: promotion of ventral migration and neuronal differentiation. 332 Development 124, 1953-62 (1997).

333 22. Schramm, A. et al. Mutational dynamics between primary and relapse neuroblastomas. 334 Nat. Genet. 47, 872-877 (2015).

335 23. Reiff, T. et al. Neuroblastoma phox $2 \mathrm{~b}$ variants stimulate proliferation and 336 dedifferentiation of immature sympathetic neurons. J. Neurosci. 30, 905-915 (2010).

337 24. Chapuy, B. et al. Discovery and characterization of super-enhancer-associated 338 dependencies in diffuse large B cell lymphoma. Cancer Cell 24, 777-790 (2013).

339 25. Trochet, D. et al. Germline mutations of the paired-like homeobox 2B (PHOX2B) gene 340 in neuroblastoma. Am J Hum Genet 74, 761-4 (2004).

341 26. Mosse, Y. P. et al. Germline PHOX2B mutation in hereditary neuroblastoma. Am J Hum 342 Genet 75, 727-30 (2004).

343 27. Coppola, E., d'Autréaux, F., Rijli, F. M. \& Brunet, J.-F. Ongoing roles of Phox2 344 homeodomain transcription factors during neuronal differentiation. Development 137, 4211$3454220(2010)$.

346 28. Ke, X.-X. et al. Phox2B correlates with $\mathrm{MYCN}$ and is a prognostic marker for 347 neuroblastoma development. Oncol. Lett. 9, 2507-2514 (2015).

348 29. Garraway, L. A. \& Sellers, W. R. Lineage dependency and lineage-survival oncogenes in 349 human cancer. Nat. Rev. Cancer 6, 593-602 (2006). 


\section{Online Methods}

\section{Neuroblastoma and hNCC cell lines}

Neuroblastoma cell lines used in this study have been previously described ${ }^{30}$. CHP-212, IMR-32, SH-SY5Y, SK-N-AS, SK-N-BE(2)C, SK-N-DZ, SK-NF-I and SK-N-SH were obtained from the American Type Culture Collection (ATCC). CLB cell lines were derived by V. Combaret (Lyon, France). The SH-EP and LAN-1 cell lines have been kindly provided by $M$. Schwab (Heidelberg, Germany) and J. Couturier (Paris, France). Lines GIMEN, N206, SJNB1, SJNB6, SJNB8, SJNB12 and TR-14 were obtained from R. Versteeg (Amsterdam, The Netherlands) and line GICAN was a kind gift from M. Ponzoni (Genova, Italy). The NB69 and NB-EBc1 cell lines were obtained from the European Collection of Authentified Cell Cultures and from the Children's Oncology Group, respectively. A first batch of SK-N-SH cells (batch 1) was used for the ChIP-seq and single cell analysis. A second batch (batch 2) was used for the evaluation of the chemotherapeutic agents. Batch 2 was enriched in adrenergic cells. Cell line authentication was performed by comparison of the genomic copy number profile calculated from the input ChIPseq data obtained using Control-FREEC ${ }^{31}$ (see below) with SNP array profile and STR profiling for ATCC cell lines. Cells were checked routinely by PCR for the absence of mycoplasma. Neuroblastoma cell lines were cultured at $37^{\circ} \mathrm{C}$ with $5 \% \mathrm{CO}_{2}$ in a humidified atmosphere in RPMI (GE Healthcare, for CLB cell lines, SH-EP, GICAN and NB69), in IMDM (Gibco) for NB-EBc1 (according to the provided conditions) or DMEM (GE Healthcare, for other cell lines), with $10 \%, 15 \%$ or $20 \%$ FCS (Eurobio) and $100 \mu \mathrm{g} / \mathrm{ml}$ penicillin/streptomycin (Gibco). Primary

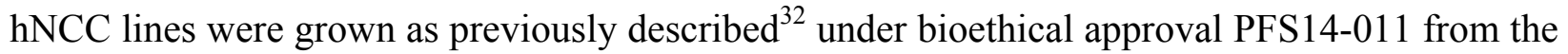
French Biomedical Agency for the use of human embryonic material to S. Zaffran. Briefly, cells were grown in Glutamax DMEM:F12 [Gibco] supplemented with 12\% FCS (Eurobio), 100 $\mu \mathrm{g} / \mathrm{ml}$ penicillin/streptomycin, $10 \mathrm{mM}$ HEPES, $100 \mathrm{ng} / \mathrm{ml}$ hydrocortisone, $10 \mu \mathrm{g} / \mathrm{ml}$ transferrin, $400 \mathrm{pg} / \mathrm{ml} \mathrm{3,3,5-thio-iodo-thyronine,} 10 \mathrm{pg} / \mathrm{ml}$ glucagon, $100 \mathrm{pg} / \mathrm{ml}$ epidermal growth factor, 1 $\mathrm{ng} / \mathrm{ml}$ insulin and $200 \mathrm{pg} / \mathrm{ml}$ fibroblast growth factor 2 (all products supplied by Sigma-Aldrich 


\section{PDX models}

383 Neuroblastoma PDXs were obtained from stage L2 (MAP-IC-A23-NB-1), stage 3 (IGR-NB8) or 384 stage 4 (IGR-N835, MAP-GR-A99-NB-1, MAP-GR-B25-NB-1 and HSJD-NB-01133). None of 385 them was related to the used cell lines. All PDXs but MAP-IC-A23-NB-1 had MYCN amplification. PDXs IGR-NB8, IGR-N835 $5^{34,35}$ were obtained using female Swiss nude mice of 6-8 weeks at engraftment whereas female NSG mice were used for MAP-GR-A99-NB-1 and MAP-GR-B25 PDXs. These PDX models are developed and maintained within the project "Development of Pediatric PDX models" approved by the experimental ethic committee 26 (CEEA26 - Gustave Roussy) under the number 2015032614359689v7. The MAP-IC-A23-NB-1 (IC-pPDX-17) and HSJD-NB-011 models were obtained using female SCID mice of 10-11 weeks or female Swiss nude mice of 3-6 weeks at engraftment. Animal studies at SJD were approved by the local animal care and use committee (Comite Etico de Experimentacion Animal at Universidad de Barcelona, protocol 135/11). All experiments were performed in accordance with European legislation. MAP-IC-A23-NB-1, MAP-GR-A99-NB-1 and MAP-GR-B25-NB-1 PDXs were obtained through the Mappyacts protocol (clinicaltrial.gov: NCT02613962).

\section{Patient samples}

Three diagnosis/relapse pairs of tumors (Pair1/2/3-Diagnosis and Relapse; all stage 4; Table S2) were studied in this work. The relapse samples were obtained through the Mappyacts protocol. The MAP-GR-B25-NB-1 PDX was derived from the relapse of pair 1. Analysis of biological material from patients, including study of expression profiles of neuroblastoma samples was 404 approved by the Institut Curie's Institutional Review Board. This study was authorized by the 405 decision of the ethics committees « Comité de Protection des Personnes Sud-Est IV », references 406 L07-95 and L12-171, "Comité de Protection des Personnes Ile de France 1", reference 0811728 407 and "Comité de Protection des Personnes Ile de France 3" reference 3272. Written informed 408 consent was obtained from parents or guardians according to national law. 


\section{ChIP-sequencing}

414 H3K27ac, PHOX2B, HAND2 and GATA3 chromatin immunoprecipitation (ChIP) was 415 performed using the iDeal ChIP-seq kit for Histones or iDeal ChIP-seq kit for Transcription 416 Factors (Diagenode) using the following antibodies: ab4729 (rabbit polyclonal, Abcam) for 417 H3K27ac, sc-376997X (mouse monoclonal), sc-9409 and sc-22206X (goat polyclonal) from 418 Santa Cruz Biotechnology for PHOX2B, HAND2 and GATA3, respectively. Ten million cells 419 were cross-linked with $1 \%$ formaldehyde for $10 \mathrm{~min}$ followed by quenching with $125 \mathrm{mM}$ 420 glycine final concentration for $5 \mathrm{~min}$ at room temperature. Chromatin was isolated by the 421 addition of lysis buffer, and lysates sonicated to obtain sheared chromatin to an average length of $422 \sim 300 \mathrm{bp}$. ChIP was performed with chromatin of 1 million cells for H3K27ac and 3.75 million 423 cells for transcription factors. The equivalent of $1 \%$ of chromatin used for TFs was kept to 424 quantify input and reverse cross-linked $4 \mathrm{~h}$ at $65^{\circ} \mathrm{C}$ with proteinase $\mathrm{K}$. ChIP was performed 425 overnight at $4^{\circ} \mathrm{C}$ on a rotating wheel with $1 \mu \mathrm{g}$ of antibody for H3K27ac, $2 \mu \mathrm{g}$ for HAND2 and 5 $426 \mu \mathrm{g}$ for PHOX2B and GATA3. Protein A-coated magnetic beads were precleared with antibodies $4273 \mathrm{~h}$ at $4^{\circ} \mathrm{C}$ only for transcription factors. After ChIP, chromatin was eluted $30 \mathrm{~min}$ at room 428 temperature and reverse cross-linked $4 \mathrm{~h}$ at $65^{\circ} \mathrm{C}$ with proteinase $\mathrm{K}$. DNA was precipitated and 429 purified with magnetic beads with the Ipure kit (Diagenode). Before sequencing, ChIP efficiency 430 was validated by quantitative PCR for each antibody on specific genomic regions using 431 powerSYBR ${ }^{\circledR}$ Green Master mix (Applied Biosystems) and compared for each primer pair to the 432 input DNA. Primers are available upon request.

433 For PDX samples, frozen tumors were reduced to powder with a pestle and then resuspended in 434 PBS. Crosslinking of chromatin was performed by adding 1\% formaldehyde for 8 min with 435 agitation on a rotating wheel. Lysis of cells, fragmentation of chromatin and ChIP were 436 performed as described above for cell lines using the iDeal ChIP-seq kit for Histones.

437 Illumina sequencing libraries were prepared from ChIP and input DNA using the TruSeq ChIP 438 library preparation kit according to the manufacturer's protocol. Briefly, DNA were subjected to 439 consecutive steps of end-repair, dA-tailing and ligation to TruSeq indexed Illumina adapters. 440 Size-selection was performed only for the H3K27ac ChIP (100 - 600 bp). After a final 441 amplification step of 14 cycles, the resulting DNA libraries were quantified using a qPCR 442 method (KAPA library quantification kit) and sequenced on the Illumina HiSeq2500 instrument 
443 (rapid run mode; single reads $100 \mathrm{nts}$ ).

ChIP-seq analysis

447 ChIP-seq reads were mapped to the human reference genome hg19/GRCh37 using Bowtie2 $448 \mathrm{v} 2.1 .0^{36}$. Low mapping quality reads $(\mathrm{Q}<20)$ were discarded; duplicate reads were kept in order 449 to detect signal in genomic amplification regions. Enriched regions (peaks) were called using 450 HMCan v1.30 37 with the following parameters: min fragment length $100 \mathrm{bp}$, median fragment 451 length $250 \mathrm{bp}$, maximal fragment length $400 \mathrm{bp}$, small bin length $50 \mathrm{bp}$, large bin length $25 \mathrm{~kb}$, 452 p-value threshold 0.05 , merging distance $200 \mathrm{bp}$, number of iterations 20, final threshold 0.1 , 453 removing duplicates: False. Regions from the hg19 ENCODE blacklist ${ }^{38}$ were excluded from the 454 analysis. HMCan output included ChIP density profiles corrected for the GC-content and copy 455 number bias (*.wig) and narrow and large enrichment regions further called peaks (*.bed). 456 Density profiles were then normalized between samples with an in-house R script based on the 457 median density values in the 5,000 highest peaks discounting the first 100 peaks as they may 458 correspond to amplification regions. Peaks with low signal (i.e., low HMCan score values) were 459 discarded (in-house script correlating peak length and peak signal, 460 https://github.com/BoevaLab/LILY/).

461 The Control-FREEC ${ }^{31}$ algorithm was applied to input samples (default parameters; input: *.bam 462 files) to obtain copy number profiles of each cell line. These profiles matched known copy 463 number profiles for these neuroblastoma cell lines.

464 To call enhancers and super-enhancers, a modified version of ROSE ${ }^{12,39}$ dubbed LILY was used 465 (http://BoevaLab.com/LILY/). First, large H3K27ac peaks were stitched together, using a default 466 distance of $12.5 \mathrm{~kb}$, while promoter regions $( \pm 2.5 \mathrm{~Kb}$ from the transcription start site) were 467 excluded. Then each region received a SE score corresponding to the sum of normalized 468 H3K27ac density values (already corrected for copy number and GC-content bias by HMCan ${ }^{37}$ ). 469 The regions were sorted according to the SE score. The threshold of the score distinguishing 470 typical enhancers from SEs was determined by ROSE. For twenty-five neuroblastoma cell lines, 471 the average number of SEs identified per cell line was 1,252 (standard deviation 385). The 472 highest number of SEs was detected in GIMEN and SH-EP (1,901 and 1,819 regions 473 respectively). 
474 ChIP-seq experiments for H3K27ac were performed once for every sample except for the CLB-

475 GA cell line for which the experiment was performed in two biological replicates. We used these 476 replicate samples to document the reproducibility of the SE calling and SE score calculation 477 (Figure S21). Among the top 500 SEs of replicate 1, 93\% were annotated as active SEs in 478 replicate 2.

479 To generate a list of neuroblastoma SEs, we superimposed the SE regions predicted in the 480 twenty-five cell lines and excluded regions shorter than $12 \mathrm{~Kb}$. In order to avoid stitching of 481 several neighboring SE regions into one, long regions with several sub-peaks were separated in 482 sub-regions using as a threshold one half of the median number of SEs. Overall, 4,336 regions 483 with overlapping SEs detected in more than one sample were annotated as putative 484 neuroblastoma SEs (Table S3). SEs were assigned to the RefSeq genes (hg19, version Sep 16, 485 2016) using the information about locations of topologically associating domains (TADs) in 486 eight human cell lines ${ }^{40}$. Among all genes located in the same TAD with a SE and therefore 487 possibly regulated by a SE, we selected these with the highest correlation between the gene 488 expression and the SE score in the 33 samples of these study (threshold 0.361 corresponding to 489 the adjusted p-value ('FDR') of 0.05, Figure S11). Of note, each gene can have several SE 490 regions and each SE can be assigned to a number of genes (Table S3). In total, neuroblastoma 491 SEs were assigned to 4,791 genes. Similarly, we detected and assigned to genes 1,639 SEs active 492 in both hNCC samples.

493 For further analysis, we kept only SE regions active in at least two neuroblastoma cell lines or 494 hNCC samples (5,975 regions). This was done to remove cell-line specific events and false 495 positive predictions of SE regions.

496 Principal Component Analysis (PCA) for 33 samples (25 neuroblastoma cell lines, 6 497 neuroblastoma PDXs and 2 hNCC lines) was performed on log2 values of SE scores of 5,975 498 SEs. Table S3 shows contributions of the SE regions to the first two principal components. 499 Analysis of samples in the first principal components suggested their separation into group I 500 (CLB-GA, CLB-MA, CLB-CAR, CLB-BER-Lud, CLB-PE, NB69, NB-EBc1, SJNB1, SJNB6, 501 SJNB8, IMR-32, LAN-1, N206, SK-N-BE(2)C, SK-N-DZ, SK-N-FI, TR14, SH-SY5Y), group II 502 (GICAN, SH-EP, GIMEN) and the intermediate group (SK-N-SH, SK-N-AS, SJNB12, CHP503 212). Table S3 includes information about fold changes and p-values for the two-sided Wilcoxon 504 test for differential analysis of SE scores between group I and II. 
To detect known transcription factor binding motifs enriched in neuroblastoma SEs (cell lines of group I and II) and SEs of hNCC, we applied the i-cisTarget ${ }^{41}$ method to the list of 2,227, 1,850 and 1,640 valley regions in H3K27ac peaks overlapping the 100 top SEs of group I and II and

508 hNCC, respectively.

509 CRC in the neuroblastoma cell lines, PDX samples and hNCC lines were detected using COLTRON ${ }^{19}$ based on the list of samples' SEs with the following properties: (i) SE score correlated with gene expression in our set of $31 \mathrm{NB}$ samples and (2) SE region was detected in more than 2 cell lines in our study. We then parsed the files with ranked cliques to see whether a given TF was predicted to be involved in a CRC of a given sample. We kept TFs present in over $50 \%$ of cell lines from group I $(n=18)$ or group II $(n=3)$. This resulted in 69 TFs. From the 515 COLTRON predictions, we excluded 17 transcription factors that were not associated with a SE in our analysis (Figure S10). As motif enrichment analysis discovered a significant enrichment in homeobox and AP-1 motifs of neuroblastoma SEs (Figures S4 and S8), among these 52 TFs, we selected those that were predicted by COLTRON to occur in the same CRC as the homeobox TF PHOX2B or AP-1 TFs (JUN, JUNB, FOSL1 or FOSL2) in more than $50 \%$ of cell lines of group I or II. This resulted in 37 TFs (Figure 1g). Clustering of the 37 genes (hclust, McQuitty method) based on the correlation of their expression defined two modules (module $1, \mathrm{n}=7$, includes 522 PHOX2B; module 2, $\mathrm{n}=15$, includes FOSL1 and FOSL2) (Figure S22).

523 Motif discovery in ChIP-seq peaks of GATA3, HAND2 and PHOX2B was performed using the 524 Position Analysis tool of the RSAT package ${ }^{42}$ (Oligonucleotide size: HAND2: 5; GATA3: 5; 525 PHOX2B: 8).

526 To calculate average ChIP-seq density profiles around the PHOX2B peak maximum positions, 527 we first extracted all 2,400 bp regions centered on PHOX2B ChIP-seq binding sites and kept 528 those that overlapped peaks of all three TFs. We obtained 14,693 such regions throughout the 529 whole human genome for the CLB-GA cell line. 2,078 out of them were located within the 500 530 strongest neuroblastoma SEs. ChIP-seq density for each TF for each region was rescaled to have 531 a maximum value of 1 corresponding to the peak maximum. We then plotted the average 532 rescaled density for the 2,078 regions.

\section{RNA-sequencing and transcriptome read alignment}

535 Total RNA was extracted from fresh cells or frozen tumors using TRIzol@ Reagent (Invitrogen), 
or AllPrep DNA/RNA Mini Kit (Qiagen) or NucleoSpin RNA kit (Macherey-Nagel; for the SK-

$537 \quad \mathrm{~N}-\mathrm{SH}$ cell line treated with chemotherapy). All samples were subjected to quality control on a 538 Bioanalyzer instrument and only RNA with RIN (RNA Integrity Number) $>6$ were used for 539 sequencing. RNA sequencing libraries were prepared from $1 \mu \mathrm{g}$ of total RNA using the Illumina 540 TruSeq Stranded mRNA Library preparation kit which allows performing a strand-specific 541 sequencing. A first step of polyA selection using magnetic beads is done to focus sequencing on 542 polyadenylated transcripts. After fragmentation, cDNA synthesis was performed and resulting 543 fragments were used for dA-tailing and then ligated to the TruSeq indexed adapters. PCR 544 amplification is finally achieved to create the final cDNA library. After qPCR quantification, 545 sequencing was carried out using 2 x 50 cycles (paired-end reads $50 \mathrm{nts}$ ) for all samples (except 546 SH-EP, 2 x 100; Pair1-Relapse and Pair3-Relapse, 2 x 75; Pair2-Relapse, 2 x 150). Sequencing 547 was performed with the Illumina HiSeq2500 instrument (high output mode) except for cases 548 Pair1-Relapse and Pair2-Relapse analyzed with the NextSeq500 instrument and Pair3-Relapse3 549 analyzed on a HiSeq4000 instrument. Reads were aligned to the human reference genome 550 hg19/GRCh37 using TopHat2 v2.0.6 ${ }^{43}$ with the following parameters: global alignment, no 551 mismatch in the $22 \mathrm{bp}$ seed, up to three mismatches in the read, library type fr-firststrand.

552 Gene expression values (FPKM=fragments per kilobase per million reads) were computed by 553 Cufflinks v2.2.1 ${ }^{44}$ and further normalization between samples was done using quantile 554 normalization (R/Bioconductor package limma) ${ }^{45}$.

\section{Western blots}

558 Western blots were carried out using standard protocols with the following antibodies: PHOX2B 559 (sc-376997 from Santa Cruz Biotechnology at 1:500) and anti-vinculin (ab18058 from Abcam at 560 1:1,000). Membranes were then incubated with an anti-mouse immunoglobulin $G(\operatorname{IgG})$ 561 horseradish peroxidase-coupled secondary antibody (1:3,000, NA931V) from GE Healthcare. 562 Proteins were detected by enhanced chemiluminescence (PerkinElmer).

564 Single cell gene expression analysis

565 Single cells loading, capture and mRNA pre-amplification were performed following the 566 Fluidigm user manual "Using $\mathrm{Cl}$ to Capture Cells from Cell Culture and Perform 
567 Preamplification Using Delta Gene Assays". Briefly, cells were dissociated using TrypLE 568 Express reagent (Gibco), washed 2 times in PBS and 2,000-4,000 cells were loaded onto a 569 medium size $(10-17 \mu \mathrm{m}) \mathrm{C} 1$ single-cell auto prep IFC (Fluidigm). The capture efficiency was 570 assessed by imaging capture sites under the microscope and cell viability was investigated with 571 ethidium homodimer-1 and calcein AM stains (LIVE/DEAD kit, Thermo Fisher Scientific). 572 Capture sites containing more than one cell or a dead cell were later excluded. Lysis, reverse 573 transcription, and specific target preamplification steps were done on the $\mathrm{C} 1$ machine according 574 to the Fluidigm user manual. Preamplification was done with inventoried pairs of unlabeled 575 primers coupled with a Taqman probe FAM-MGB (Applied Biosystems TaqMan Gene 576 Expression Assays, Thermo Fischer Scientific) for each of the module 1 and 2 genes and 4 577 housekeeping genes (GAPDH, ACTG1, ACTB and RPL15). Preamplification products were 578 harvested and high throughput real-time PCR was performed using the Fluidigm Biomark HD 579 system with 48.48 gene expression Dynamic Arrays. For each cell line, a bulk control 580 representative of 400 cells was processed the same way as the single cells. The raw data were 581 first analyzed with the Fluidigm Real-Time PCR Analysis Software and exported to csv files for 582 further analysis.

583 Gene expression value was normalized using the geometric mean of all 4 housekeeping genes $\mathrm{Ct}$ 584 values of a given cell, cells were excluded if this geometric mean was $>16.5$. The Livak method

$585\left(2^{-\Delta \Delta \mathrm{Ct}}\right)$ was applied using the gene expression values of the SK-N-SH cell line population as 586 reference for the relative expression. Hierarchical clustering was performed using one minus 587 Pearson correlation with an average linkage method including heatmap using Morpheus platform 588 (https://software.broadinstitute.org/morpheus).

589

590 Treatment of cell lines with chemotherapy

591 SH-EP and SH-SY5Y cell lines were plated in 96-well plates two days before the addition of 592 cisplatin, etoposide or doxorubicin. Seeding densities for each cell lines were optimized to reach $59380 \%$ of confluency in the untreated cells. Cells were treated with chemotherapeutic agents for 48 594 h. Cell viability was then measured using the in vitro Toxicology Assay Kit, Resazurin-based, 595 following manufacturer's instructions (Sigma-Aldrich).

596 SK-N-SH cells were plated in 6-well plates and then treated with cisplatin $(7.5 \mu \mathrm{M})$ or 
doxorubicin $(100 \mathrm{nM})$ for 7 days, medium and drugs were changed every 2 days. RNAs were 598 extracted using NucleoSpin RNA kit (Macherey-Nagel).

\section{Doxycycline-inducible shRNA systems}

600 PHOX2B-specific short hairpin RNAs sh1783 (5'-CCGGTGGAAGGCAGAAACCATTAAA601 CTCGAGTTTAATGGTTTCTGCCTTCCATTTTTG-3') and sh1437 (5'-CCGGAGTAATCG602 CGCTAAGAATAAACTCGAGTTTATTCTTAGCGCGATTACTTTTTTG-3') were selected 603 from Sigma Mission shRNA library and cloned into the pLKO-Tet-On all-in-one system $^{46}$ 604 (Addgene). Lentiviral particles were produced in HEK293T cells and CLB-GA cells were 605 infected as previously described ${ }^{4}$. SH-SY5Y cells were incubated with viral particules for 48 606 hours without polybrene. Selection with puromycin (Invitrogen) at $400 \mathrm{ng} / \mathrm{ml}$ or $1 \mu \mathrm{g} / \mathrm{ml}$, 607 respectively, was performed $24 \mathrm{~h}$ after infection and maintained during all culture experiments, 608 for CLB-GA and SH-SY5Y cells, respectively. PHOX2B knockdown efficacy was assessed by 609 Western blot $24 \mathrm{~h} / 48 \mathrm{~h} / 96 \mathrm{~h}$ after the addition of doxycycline (100 $\mathrm{ng} / \mathrm{ml}$ or $1 \mu \mathrm{g} / \mathrm{ml})$. For colony 610 formation assays, $6 \times 10^{4}$ transduced cells were plated at day 0 in 6-well dishes and stained with 611 crystal violet at day $11^{47}$.

612 Proliferation assays

613 Cells were counted in real-time with an $\mathrm{xCELLigence}^{\mathrm{TM}}$ instrument (ACEA Biosciences) 614 monitoring imped-ance across gold microelectrodes. $10^{4}$ infected CLB-GA or SH-SY5Y cells 615 were seeded per well of a 96-well plate in $200 \mu 1$ medium containing doxycycline at $100 \mathrm{ng} / \mathrm{ml}$ 616 or $1 \mu \mathrm{g} / \mathrm{ml}$ (quintuplicates per group) or no doxycycline. Medium was refreshed after $48 \mathrm{~h}$. For 617 cell counting, $2 \times 10^{4}$ infected CLB-GA or $10^{5}$ infected SH-SY5Y were plated in 24-well plates in 618 the presence or absence of doxycycline at $100 \mathrm{ng} / \mathrm{ml}$ or $1 \mu \mathrm{g} / \mathrm{ml}$. The number of living cells was 619 counted at day 4, 7, 10 and 14 (triplicates per group) for CLB-GA and at day 8 ( $\mathrm{n}=5 / 6$ technical 620 replicates) for SH-SY5Y cells using a Vi-cell XR Cell Viability Analyzer (Beckman Coulter)

623 Statistical analysis

624 To calculate p-values for Pearson correlation (null hypothesis consisted in zero Pearson 625 correlation), we implemented a one-sided permutation test. Number of permutations was $10^{4}$ 
626 when calculating p-values for correlation between SE score and gene expression (Figure S11)

627 and $10^{6}$ in the test for correlation between gene expression of the noradrenergic and NCC-like 628 modules in the set of tumors (Figure 2). This test does not need the data to follow the normal 629 distribution and does not require equal variation between the groups that are statistically 630 compared.

631

632 Xenotransplantation experiments and mice

$63310 \times 10^{6}$ CLB-GA cells transduced with the shRNA against PHOX2B (sh1783) were injected 634 subcutaneously in the flanks of 6-week-old NSG mice (Charles Rivers Laboratories) in an equal 635 mix of PBS and Matrigel (BD Biosciences). When tumors reached a volume of around $170 \mathrm{~mm}^{3}$, 636 mice were randomly assigned to the control (5\% sucrose in drinking water) or the treatment 637 (doxycycline $(2 \mathrm{mg} / \mathrm{l})$ and 5\% sucrose in drinking water) groups. Tumor growth was monitored 638 with a caliper every day. Mice were killed once tumors reached a volume of around 3,000 $\mathrm{mm}^{3}$ 639 calculated as $\mathrm{V}=\mathrm{a} / 2 \times \mathrm{b} \times((\mathrm{a}+\mathrm{b}) / 2)$ with a being the largest diameter and $\mathrm{b}$ the smallest. 640 Experiments were conducted in accordance with the recommendations of the European 641 Community (86/609/EEC), the French Competent Authority, and UKCCCR (guidelines for the

642 welfare and use of animals in cancer research). Approval for this study was received from 643 Ministère de l'Education Nationale, de l'Enseignement Supérieur et de la Recherche 644 (authorization number 5524-20 1605311607151 v5).

645

\section{PHOX2B immunohistochemistry}

647 Mice tumors were fixed with acidified formal alcohol (AFA) for $24 \mathrm{~h}$ and paraffin-embedded. 648 Labelling was performed on $4 \mu \mathrm{m}$ sections with the BOND-III instrument (Leica Microsystems) 649 using the Bond Polymer Refine Detection ${ }^{\mathrm{TM}}$ (Leica) kit. Briefly, sections were deparaffinized, 650 antigen retrieval was performed with an EDTA-based solution (Leica) for 20 minutes at pH 9, 651 and sections were stained with a rabbit polyclonal anti-PHOX2B antibody (Abcam, EPR14423, $6521 / 1000)$.

653

654 siRNA and growth assays

655 HAND2 and GATA3 knockdown was performed with 20 nM siRNA (Hs_HAND2_3 656 \#SI00131915, Hs_HAND2_6 \#SI03046736, Hs_GATA3_7 \#SI04202681 and Hs_GATA3_8 
\#SI04212446; Control siRNA \#1027281; Qiagen) using RNAimax transfection reagent (Thermo

658 Fisher Scientific). The number of living cells was counted using a Vi-cell XR Cell Viability

659 Analyzer (Beckman Coulter) ( $\mathrm{n}=5$ or 6 technical replicates).

660

\section{Data Availability}

662 Raw data for cell line ChIP-seq and RNA-seq, and processed data for the cell lines, tumors and 663 PDXs are available in Gene Expression Omnibus (GEO) under accession number GSE90683. 664 Raw data for PDX ChIP-seq and RNA-seq will be available through EGA, as well as RNA-seq 665 data for patient samples.

666 Reviewers can access to the GEO submission using this link:

667 https://www.ncbi.nlm.nih.gov/geo/query/acc.cgi?token=khcvkaailpulxqt\&acc=GSE90683.

\section{Code availability}

669 The code of the pipeline for the SE detection from cancer ChIP-seq data is available at 670 http://boevalab.com/LILY/ .

673 30. Schleiermacher, G. et al. Combined 24-color karyotyping and comparative genomic 674 hybridization analysis indicates predominant rearrangements of early replicating chromosome 675 regions in neuroblastoma. Cancer Genet Cytogenet 141, 32-42 (2003)

676 31. Boeva, V. et al. Control-free calling of copy number alterations in deep-sequencing data 677 using GC-content normalization. Bioinformatics 27, 268-9 (2011).

678 32. Etchevers, H. Primary culture of chick, mouse or human neural crest cells. Nat. Protoc. 6, $679 \quad 1568-1577$ (2011).

680 33. Monterrubio, C. et al. Targeted drug distribution in tumor extracellular fluid of GD2681 expressing neuroblastoma patient-derived xenografts using SN-38-loaded nanoparticles 682 conjugated to the monoclonal antibody 3F8. J. Control. Release 255, 108-119 (2017).

683 34. Vassal, G. et al. Therapeutic activity of CPT-11, a DNA-topoisomerase I inhibitor, against 684 peripheral primitive neuroectodermal tumour and neuroblastoma xenografts. Br. J. Cancer 74, $685 \quad 537-545$ (1996).

686 35. Bettan-Renaud, L., Bayle, C., Teyssier, J. R. \& Benard, J. Stability of phenotypic and 
687 genotypic traits during the establishment of a human neuroblastoma cell line, IGR-N-835. Int. J. 688 Cancer 44, 460-466 (1989).

689 36. Langmead, B. \& Salzberg, S. L. Fast gapped-read alignment with Bowtie 2. Nat. Methods 9, $690 \quad 357-359$ (2012).

691 37. Ashoor, H. et al. HMCan: a method for detecting chromatin modifications in cancer samples 692 using ChIP-seq data. Bioinformatics 29, 2979-2986 (2013).

693 38. ENCODE Project Consortium. An integrated encyclopedia of DNA elements in the human 694 genome. Nature 489, 57-74 (2012).

695 39. Lovén, J. et al. Selective inhibition of tumor oncogenes by disruption of super-enhancers. 696 Cell 153, 320-334 (2013).

697 40. Rao, S. S. P. et al. A 3D map of the human genome at kilobase resolution reveals principles 698 of chromatin looping. Cell 159, 1665-1680 (2014).

699 41. Herrmann, C., Van de Sande, B., Potier, D. \& Aerts, S. i-cisTarget: an integrative genomics 700 method for the prediction of regulatory features and cis-regulatory modules. Nucleic Acids Res. 701 40, e114 (2012).

702 42. Medina-Rivera, A. et al. RSAT 2015: Regulatory Sequence Analysis Tools. Nucleic Acids 703 Res. 43, W50-56 (2015).

704 43. Kim, D. et al. TopHat2: accurate alignment of transcriptomes in the presence of insertions, 705 deletions and gene fusions. Genome Biol. 14, R36 (2013).

706 44. Trapnell, C. et al. Transcript assembly and quantification by RNA-Seq reveals unannotated 707 transcripts and isoform switching during cell differentiation. Nat. Biotechnol. 28, 511-515 708 (2010).

709 45. Ritchie, M. E. et al. limma powers differential expression analyses for RNA-sequencing and 710 microarray studies. Nucleic Acids Res. 43, e47 (2015).

711 46. Wiederschain, D. et al. Single-vector inducible lentiviral RNAi system for oncology target 712 validation. Cell Cycle 8, 498-504 (2009).

713 47. Franken NAP, Rodermond HM, Stap J, Haveman J, van Bree C. Clonogenic assay of cells in 714 vitro. Nat Protoc. 2006; 1:2315-2319. 


\section{Acknowledgements}

This work was supported by grants from Institut Curie, Inserm, the Ligue Nationale Contre le

722 Cancer (Equipe labellisée), the Société Française de Lutte contre les Cancers et les Leucémies de 723 l'Enfant et l'Adolescent, the Institut National du Cancer (PRT-K14-061), the PHRC IC 2007-

724 2009) and by the following associations: Association Hubert Gouin, Les Bagouz à Manon, les

725 amis de Claire, Courir pour Mathieu, Dans les pas du Géant, Olivier Chape. The Mappyacts 726 protocol is supported by the PRHC, the Fondation ARC, the Société Française de Lutte contre 727 les Cancers et les Leucémies de 1'Enfant et 1'Adolescent (Fondation Enfants \& Santé), the 728 Fondation AREMIG and the Association Thibault BRIET). High-throughput sequencing has 729 been performed by the ICGex NGS platform of the Institut Curie supported by the grants ANR10-EQPX-03 (Equipex) and ANR-10-INBS-09-08 (France Génomique Consortium) from the Agence Nationale de la Recherche ("Investissements d'Avenir" program), by the Canceropole Ile-de-France and by the SiRIC-Curie program -SiRIC Grant "INCa-DGOS- 4654". Biomark analysis was done using the High Throughput qPCR-HD-Genomic Paris Centre platform supported by grants from "Région Ile-de-France". G.S. is supported by the Annenberg foundation. V.B. is supported by the ATIP-Avenir Program, the ARC Foundation (grant ARC RAC16002KSA - R15093KS), Worldwide Cancer Research Foundation (grant WCR16-1294 738 French Government through its "Investissement d'Avenir" program operated by The French 739 National Research Agency (ANR) (grant ANR-11-IDEX-0005-02). H.R. is supported by the

740 Mayent-Rothschild program from Institut Curie and the Wilhelm-Sander-Stiftung. The 741 laboratory of T.G.P.G. is supported by LMU Munich's Institutional Strategy LMUexcellent 742 within the framework of the German Excellence Initiative, the 'Mehr LEBEN für krebskranke 743 Kinder - Bettina-Bräu-Stiftung', the Walter Schulz Foundation, the Wilhelm-Sander-Stiftung 744 (2016.167.1), and by the German Cancer Aid (DKH-111886 and DKH-70112257). We are 745 grateful to M. Ponzoni (Genova, Italy) for providing the GICAN cell line, F. Tirode and C. 746 Kamoun for help with RNA-seq analysis and alignment of NGS data, respectively, O. Blanchard

747 for help in cell culture experiments and M. Caly for PHOX2B immunohistochemistry. We are 748 grateful to the animal facilities team, the Experimental Pathology Department and the Plateforme 


\section{Competing financial interests}

777 The authors declare no competing financial interests. Basse-Normandie) for providing tumor patient samples.

\section{Author information}

\section{Contributions}

Génomique of Institut Curie. We thank N. Clément, T. Adam-de-Beaumais and B. Mallon for their help in the identification of neuroblastoma diagnosis/relapse pairs and V. Bernard for pairs validation. We thank V. Saint-André for scientific discussion, J. Maliash-Planchon and the Unité de Génétique Somatique for preparation of patient samples. We thank Pr. Figarella-Branger (BB033-00097, CRB AP-HM, CRB TBM, AC-2013-1786), M. Clapisson (CRB Centre Léon Bérard, AC-2008-101), Dr Minckes, Dr Blanc-Fournier and N. Rousseau (CHU, Tumorothèque - Caen

V.B. and I.J-L. conceived the study, analyzed the data and wrote the manuscript. V.B. coordinated bioinformatics analysis and I.J.-L. coordinated the whole study. C.L. performed in vitro experiments and all ChIP experiments and participated in the study design. A.P. generated and analyzed the doxycycline-inducible anti-PHOX2B shRNA cell lines. S.D. performed the single cell analysis and study of chemotherapeutic agents. C.P.-E. performed the in vivo experiments and contributed in vitro experiments.V.R. performed all sequencing experiments. H.E. and S.T. provided hNCC cell lines and V.C. provided neuroblastoma cell lines. A.L. performed alignment of RNA-seq and ChIP-seq data. E.D.-D., B.G., D.S. and A.M.C. provided neuroblastoma PDXs. I.M. performed the reproducibility analysis. E.D. and B.D. generated the Biomark data. M.F.O. and T.G.P.G. generated lentiviral particles and provided help with lentiviral infections. S.B. coordinated and supervised sequencing experiments. G.S. participated in the study design and provided the in-house pairs of diagnosis/relapse samples with the help of E.L., G.P. and B.G. S.G.-L. participated in RNA-seq analysis. E.B. provided computational infrastructure and data storage. H.R. and T.D. provided expertise in sympathetic nervous development and transcription factors. I.J.-L and O.D. provided laboratory infrastructure. I.J.-L, V.B. and O.D. provided financial support. All authors read and approved the final manuscript. 


\section{Corresponding authors}

780 Correspondence to:

- Valentina Boeva: valentina.boeva@inserm.fr

- Isabelle Janoueix-Lerosey: janoueix@curie.fr

\section{Extended data figures and tables}

Supplementary Table 1: $M Y C N, A L K$ and $P H O X 2 B$ status of neuroblastoma cell lines and PDXs. Amp., amplification; Non amp., non amplified; WT, wild-type. NA: not available. * As determined by Sanger sequencing of exons 23 and 25.

$799 \quad$ \# In frame deletion in the second PolyAlanine track; functional change unknown.

Supplementary Table 2: Patient clinical data.

802

803 Supplementary Table 3: Characteristics of neuroblastoma and hNCC super-enhancers. Group I: 804 all neuroblastoma cell lines with the exception of SH-EP, GIMEN, GICAN, SK-N-AS, SJNB12, 805 SK-N-SH and CHP-212; Group II: SH-EP, GIMEN and GICAN.

807 Supplementary Table 4: Supervised analysis of SE scores according to MYCN status. 
809 Supplementary Table 5: Supervised analysis of SE scores according to ALK status.

810

811 Supplementary Table 6: Raw Ct values measured for housekeeping genes (GAPDH, ACTG1, 812 ACTB, RPL15) and TFs of modules 1 and 2 for single cells of the SK-N-AS, SH-EP, SH-SY5Y 813 and SK-N-SH cell lines using the Fluidigm Biomark HD.

814

815 

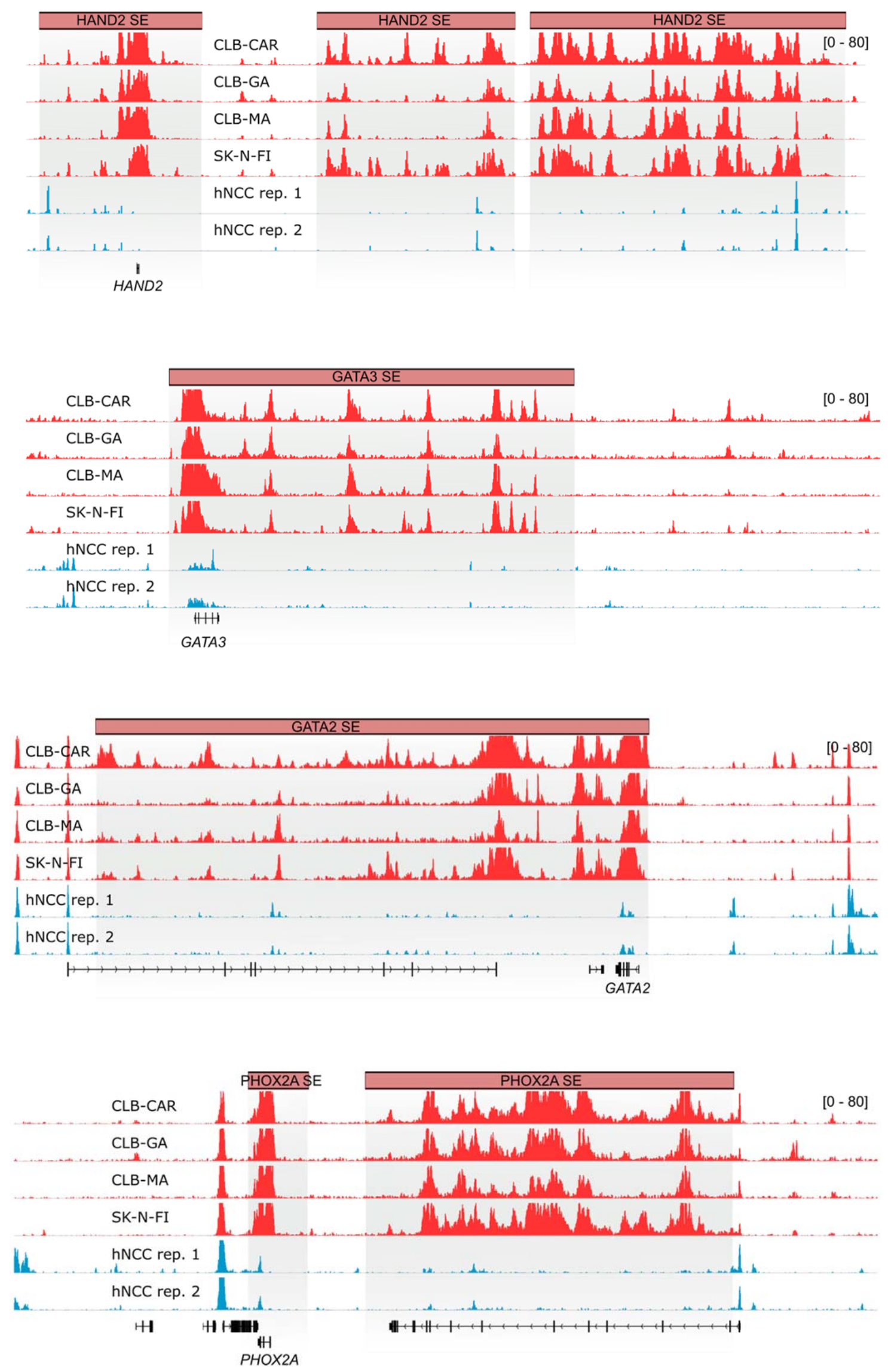
817 Supplementary Figure 1: Tracks for ChIP-seq profiles of H3K27ac at HAND2, GATA3, 818 GATA2, and PHOX2A SEs.

819

820

821

822

823

824

825

826

827

828

829

830

831

832

833 
a

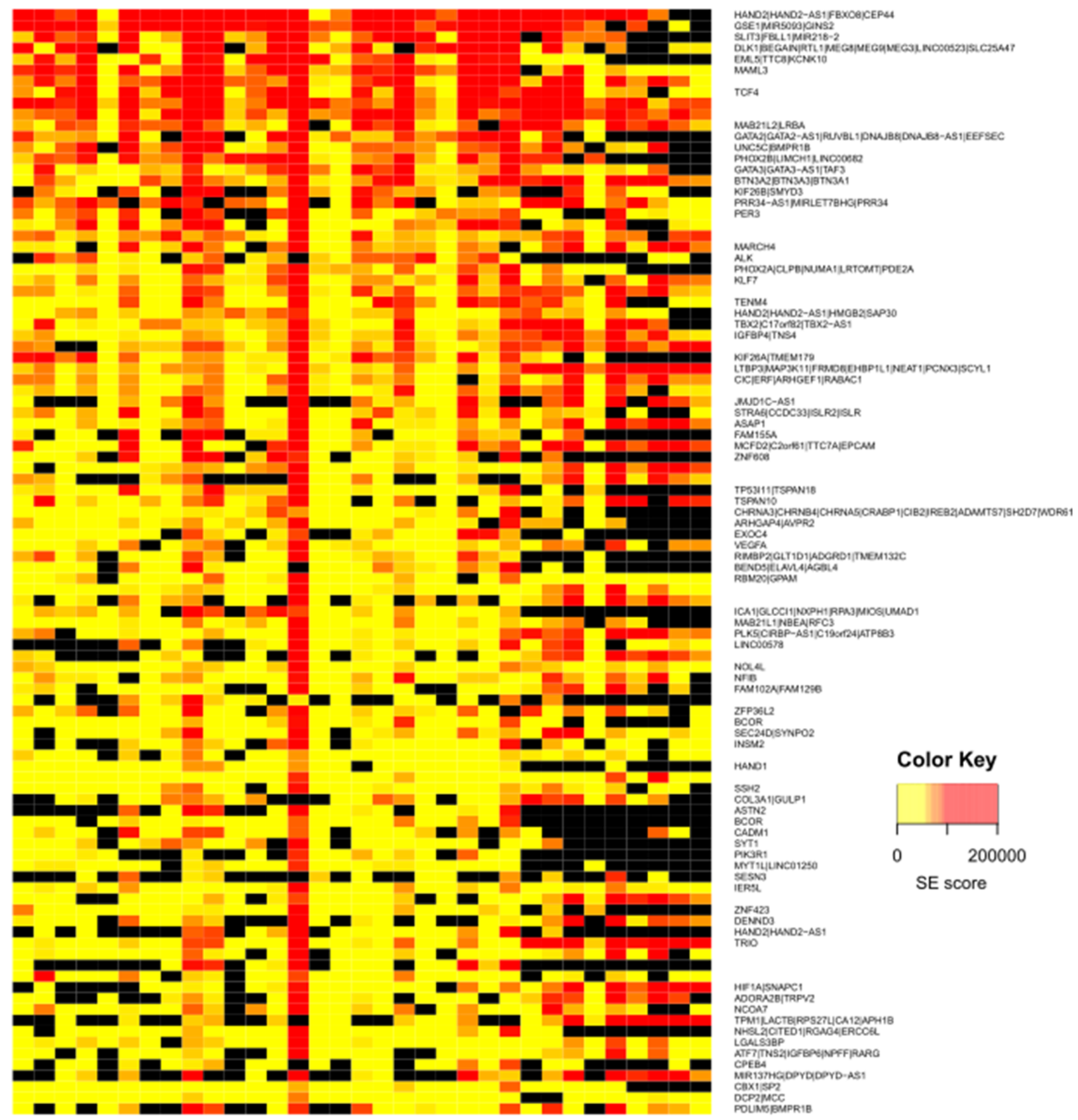

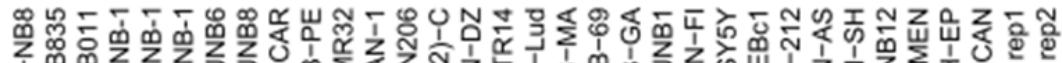

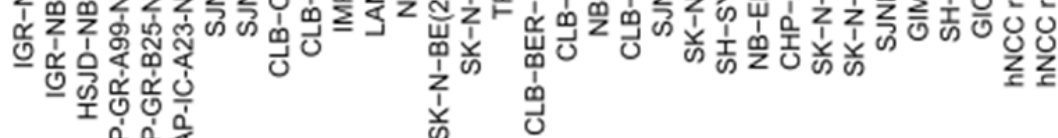

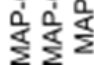


b

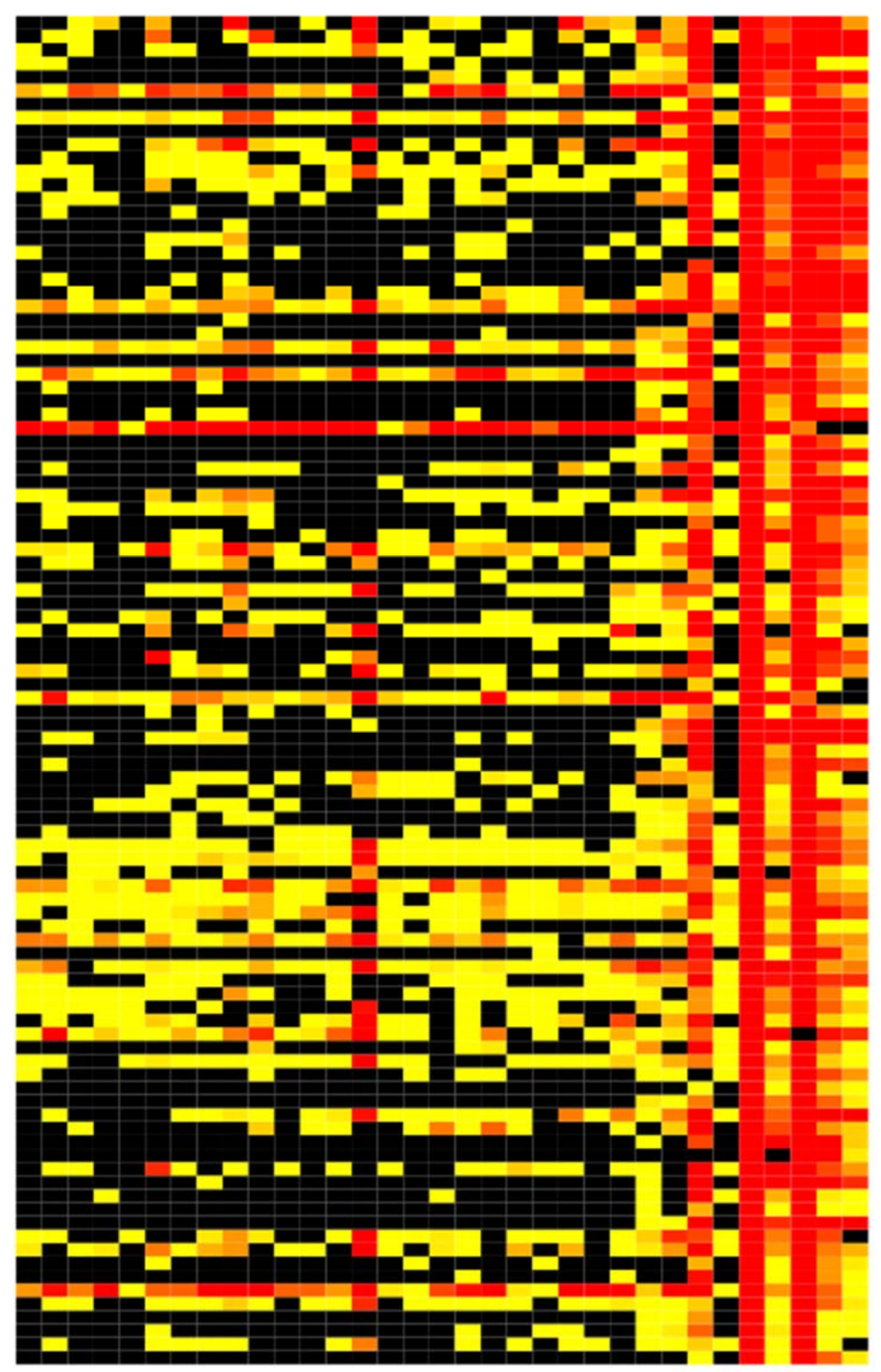

\section{Associated genes}

MRismapergopero-As!

Mechizpromecalct

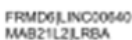

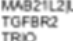

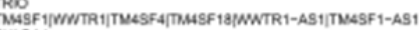

Bevoseacis

Csporyentumod

FNilunconeor

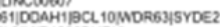

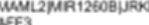

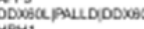

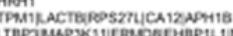

INEATIPCNoOscn,

CPMAINC-PINTCPAS

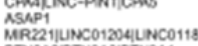

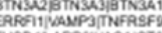

THSOMLAFOSILCACTE:

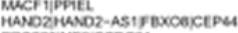

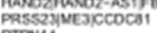

PTPN14

OCL6LPP-AS2LPP

NTC

Meccis

PUACicionts:

\section{Color Key}

QCAR3 3

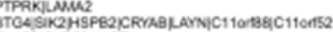

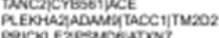

LOTUMOTONIJTFap1!

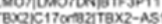

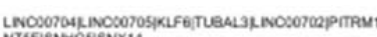

MRSOSHCLNCDCESOMRSOYNOSPD1|PUC1|FNA1ZZ

GDDDSNGMGIZZM SGNG12-AS!

YAPI ENC $39 M$ W

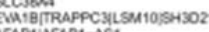

SPALIIIROSE

คMW

SRRPI

GLis3

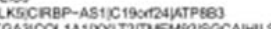

ZETESG|FuF?

TSPANito

PCDSOCDC10

FOSLNT15IOTO

HEFAS

ctornted

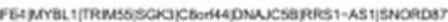

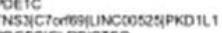

POGFCICRBCTSO

MR211UNCO1204UNCO1186

NDORLERTRPA2

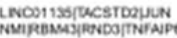

SHSAF 1

s+20us

cCocagcions2yetranasos

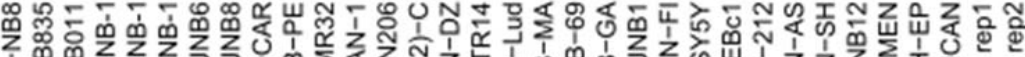
$\begin{array}{lll} & \\ 0 & \end{array}$

836 Supplementary Figure 2: Heatmap of the SE scores for the 6 PDX, 25 NB cell lines and hNCC

837 cells: top SEs of group I (a); top SEs of group II (b); SE is not detected (black). 


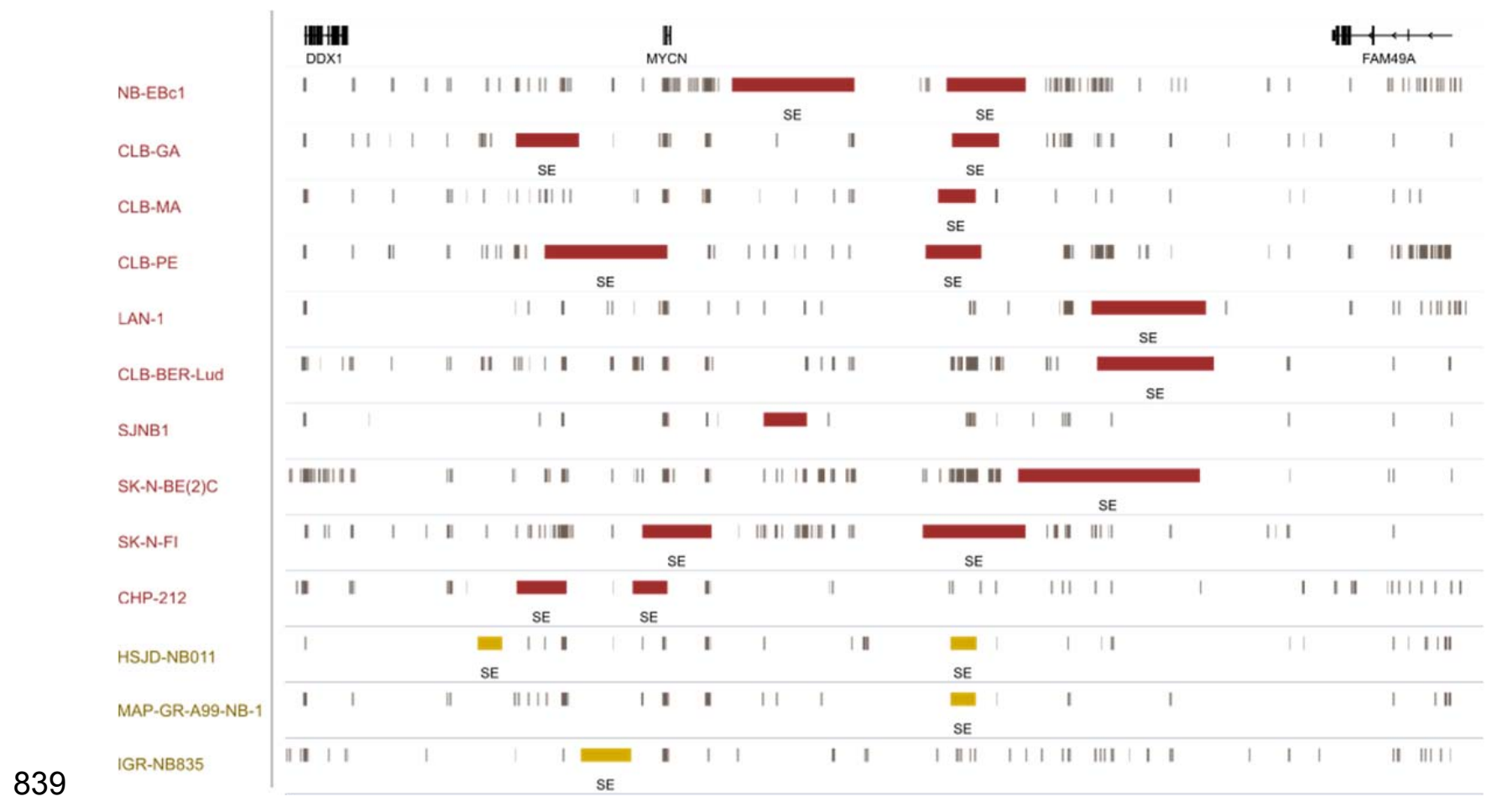

840

841

842 Supplementary Figure 3: Locations of SE regions predicted for the MYCN locus; SEs in NB 843 cell lines (red); SEs in PDX (yellow); typical enhancers and active promoters (grey). 
\# Feature

1. TAAYYNAATTA-PROP1-full

2. PGAM2

3. TAATYYAATTA-PHOX2B-DBD

4. GATA1, GATA2, GATA3, GATA4, GATA5, GATA6

5. $\mathrm{ZCCHC} 14$

6. WT1

7. TAATYYAATTA-PHOX2A-DBD

8. EGR1

9. TAATYTAATTA-PRRX1-full

10. SP1:SP3

11. SP4

12. ALX4
NES

Logo

Recovery Curve

4.21

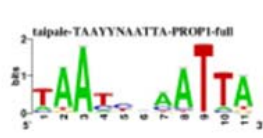

3.98
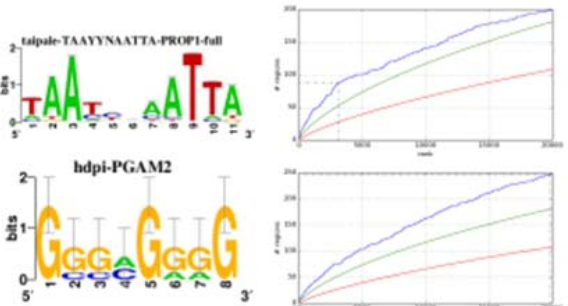

3.98

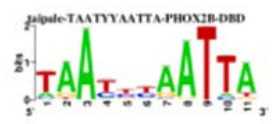

3.94

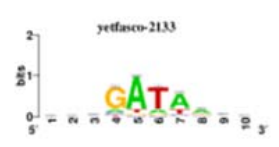

3.85
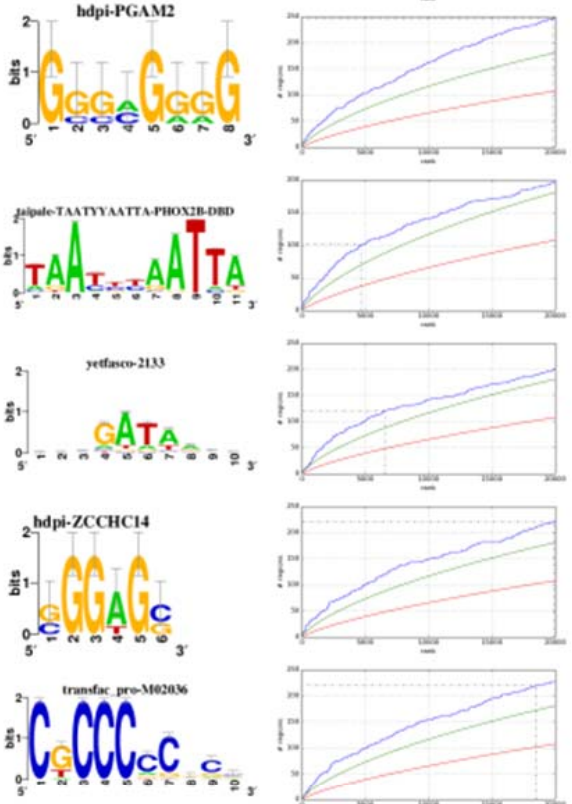

3.84

3.76
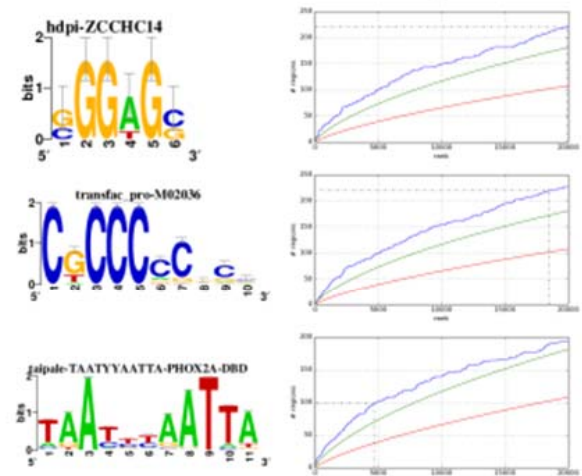

3.84
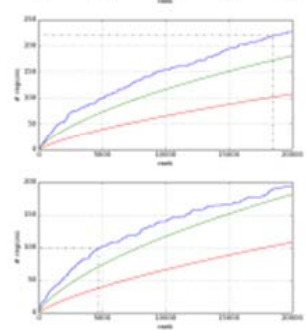

3.75
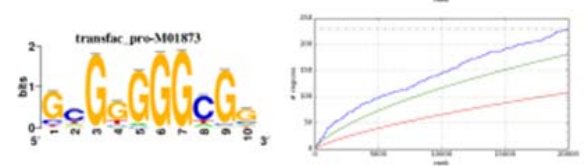

3.74
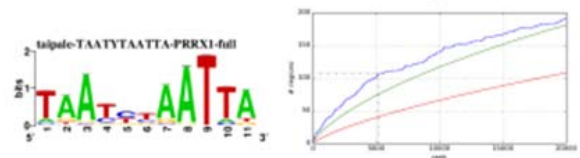

3.72
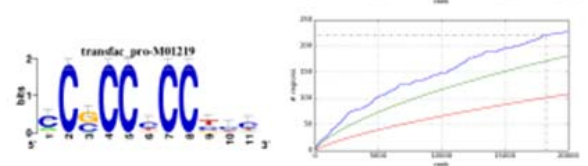

3.72
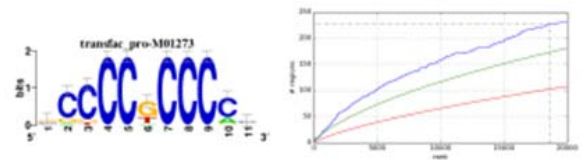

3.71
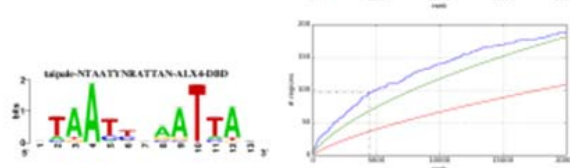

Supplementary Figure 4: i-cis Target summary (database v3.0) on H3K27ac peak valleys of the top 100 strongest SEs identified in group I. NES, Normalized enrichment score. NES threshold 3.7. NES and recovery curves are explained at the i-cisTarget website: 
849 https://gbiomed.kuleuven.be/apps/lcb/i-cisTarget/.

850

851

852

853

854

855

856

857

858

859 


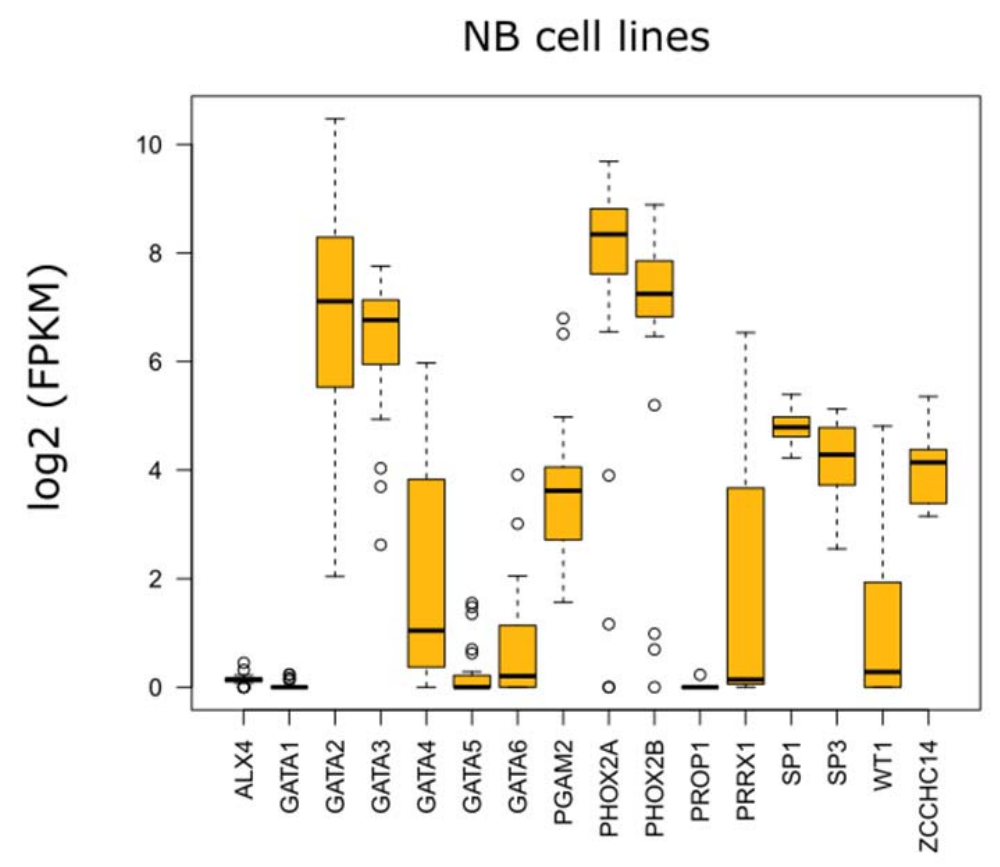

NB tumors

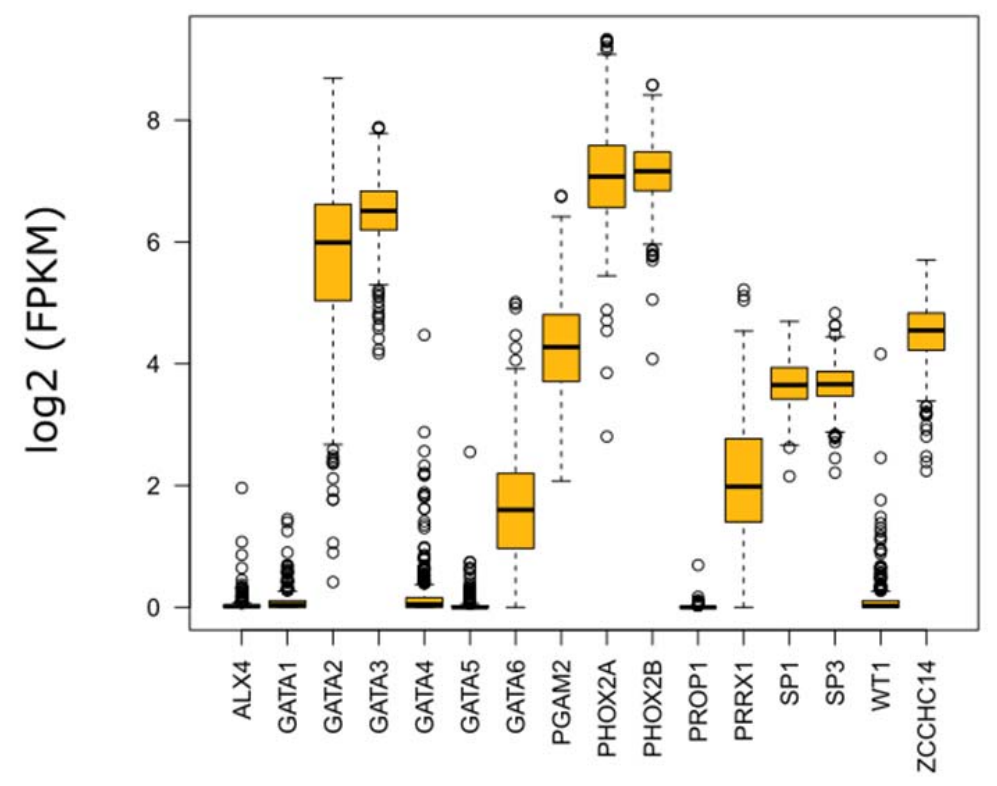

861 Supplementary Figure 5: Log2 FPKM expression values for genes coding for TFs whose 862 binding motifs are enriched in valleys of H3K27ac peaks of the top 100 strongest SEs identified 863 in group I, in neuroblastoma (NB) cell lines (our data) and NB primary tumors (498 tumors, 864 dataset GSE49711). The box represents the middle 50\% of values; the black line inside the box 865 indicates the median. 


\section{PHOX2B}

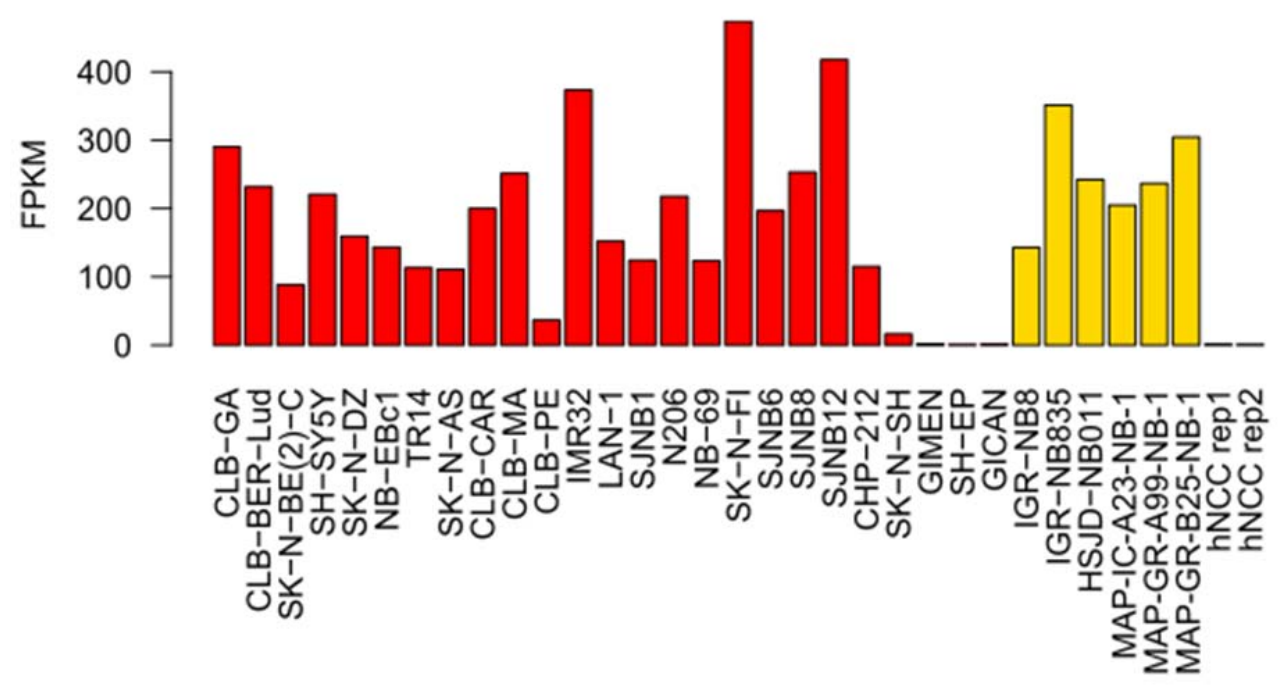

MYC

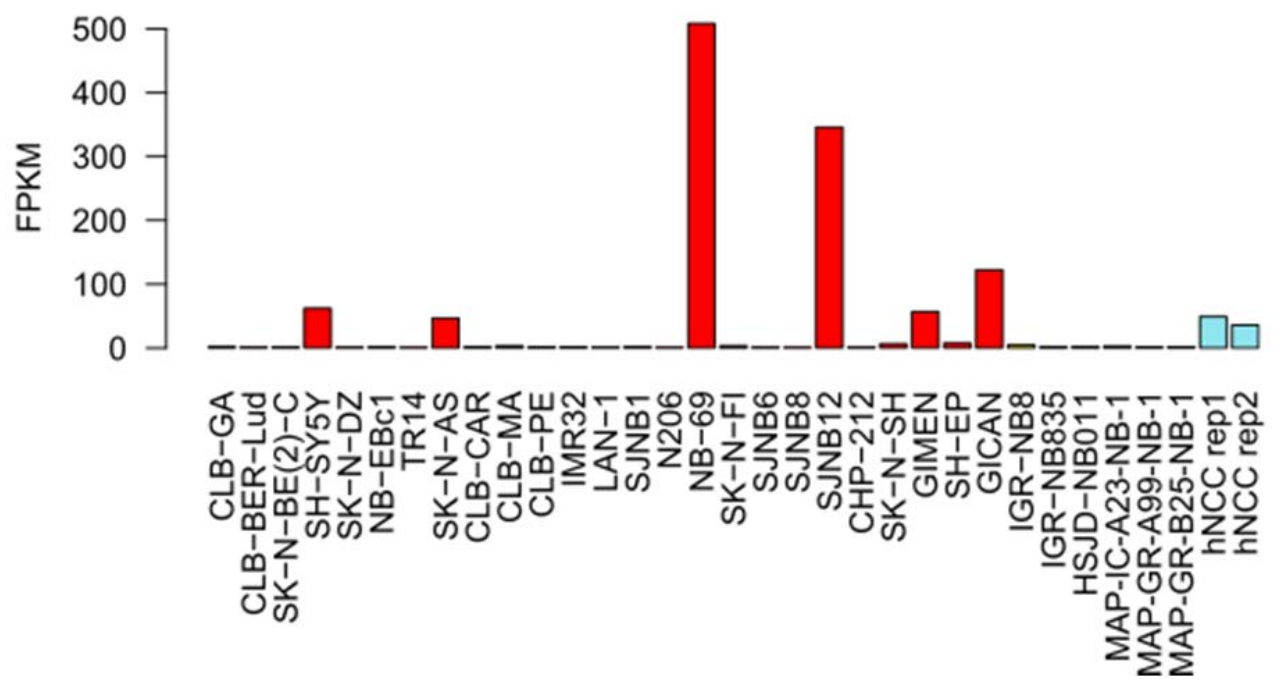

867 Supplementary Figure 6: $P H O X 2 B$ and $M Y C$ expression levels measured by RNA-seq in 868 neuroblastoma cell lines (red), PDX (yellow) and hNCC lines (blue).

869

870 


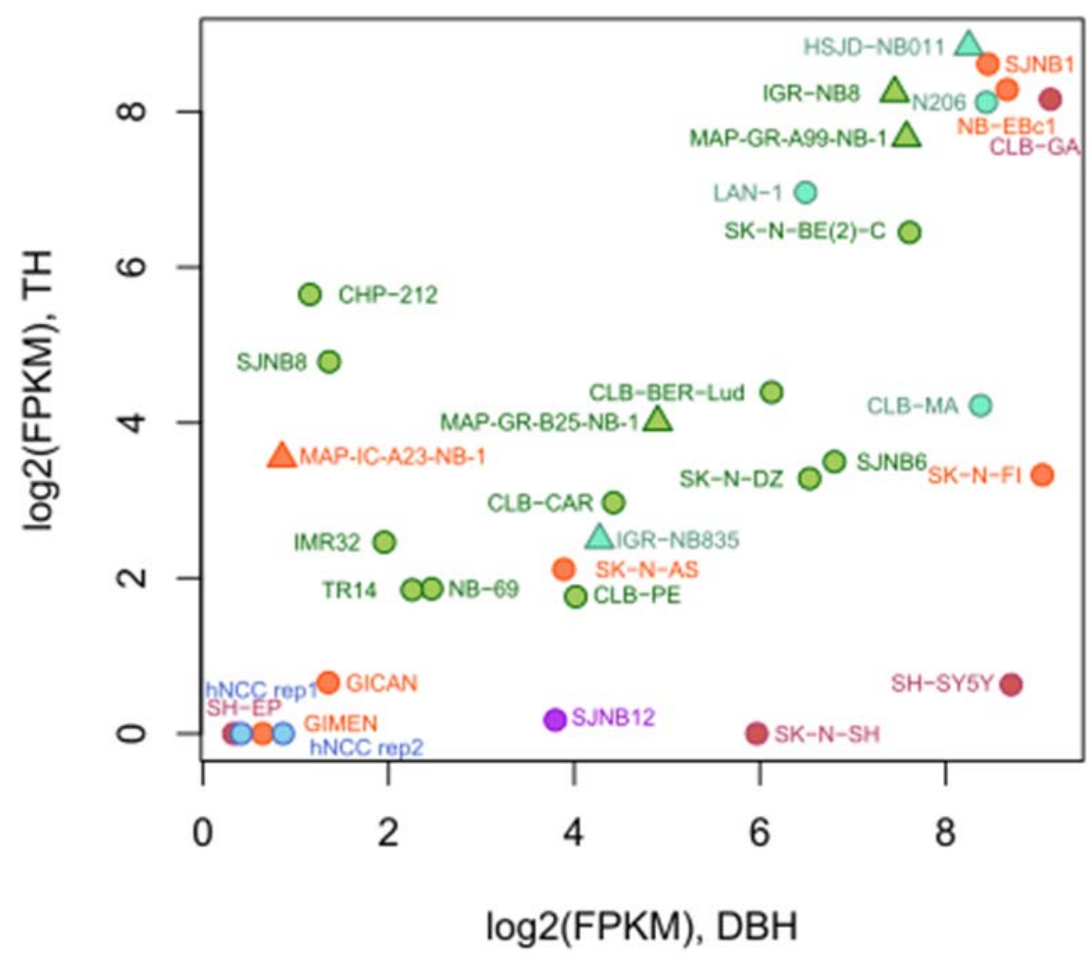

$\begin{array}{ll}\triangle \text { PDX } & \bullet \text { ALKmut } \\ \bigcirc \text { Cell line } & \bullet \text { ALKwt } \\ \bigcirc \text { MNAALKmut } & \bullet \text { MYC-amp ALKwt } \\ \circ \text { MNAALKwt } & \circ \text { hNCC }\end{array}$

874

875 Supplementary Figure 7: $\log 2$ FPKM expression values of $D B H$ and $T H$ in hNCC and 876 neuroblastoma cell lines and PDX measured by RNA-seq. 
\# Feature

1. JUN

2. AP-1: BATF, SPI1, FOS, JUNB, JUN, JUND, FOSL1, FOSL2 6.95

3. ARNT

4. FOS

5. JUNB

6. FOSL1

7. FOSB, FOS, FOSL1, FOSL2
7.12
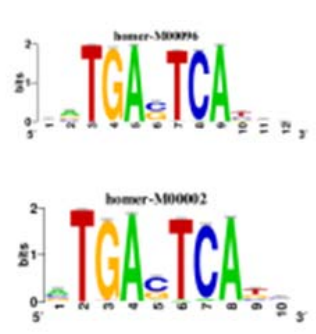

6.88
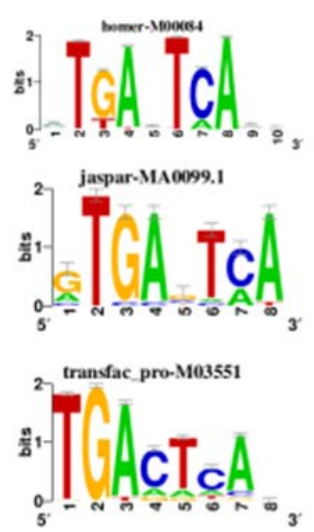

6.38

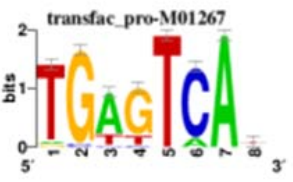

6.23

885

886

887 Supplementary Figure 8: i-cis Target summary (database v3.0) on H3K27ac peak valleys of the 888 top 100 strongest SEs identified in group II. NES, Normalized enrichment score. NES threshold 889 6.2. NES and recovery curves are explained at the i-cisTarget website: 890 https://gbiomed.kuleuven.be/apps/lcb/i-cisTarget/. 
1. BATF, FOS, JUNB, JUN, JUND, FOSL1, FOSL2

2. FOS, JUNB, FOSL1, JUND, FOSB

4.00

3. FOS

3.99

4. FOSB, FOS, FOSL1, FOSL2

5. $\mathrm{BACH} 2$

4.11

3.80

3.88
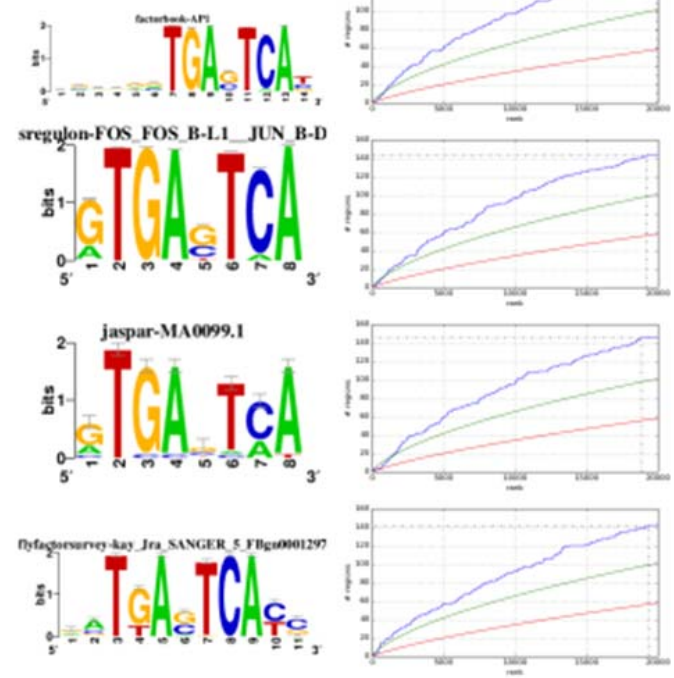

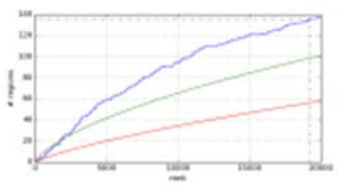

6. $E Z R$

3.71

Supplementary Figure 9: i-cis Target summary (database v3.0) on H3K27ac peak valleys of the

894 3.7. AUC threshold 0.015. NES and recovery curves are explained at the i-cisTarget website:

895 https://gbiomed.kuleuven.be/apps/lcb/i-cisTarget/.

896

897

898 


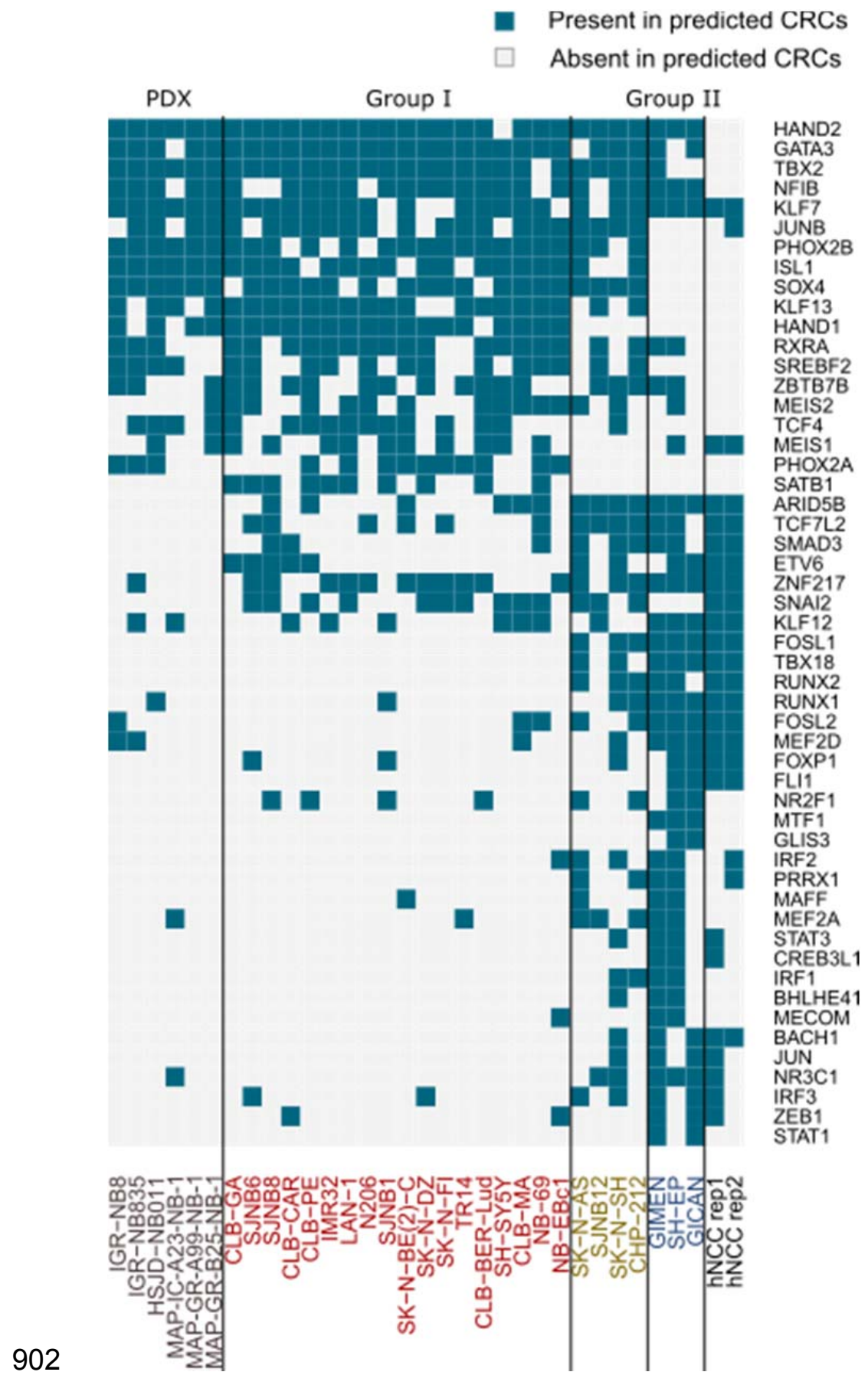

904 Supplementary Figure 10: TFs predicted to participate in CRCs in the two groups of 905 neuroblastoma cell lines, and the primary hNCC. 
a
Correlation between gene expression (FPKM) and SE strength in log scale $(n=267)$

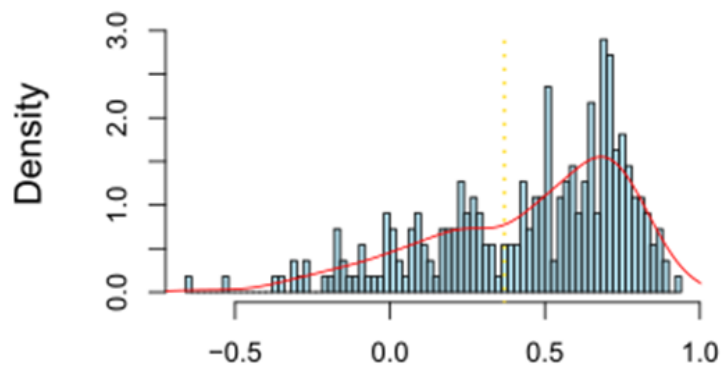

Pearson correlation coefficient b
Correlation test ajusted p-value between
gene expression and SE strength $(n=267)$

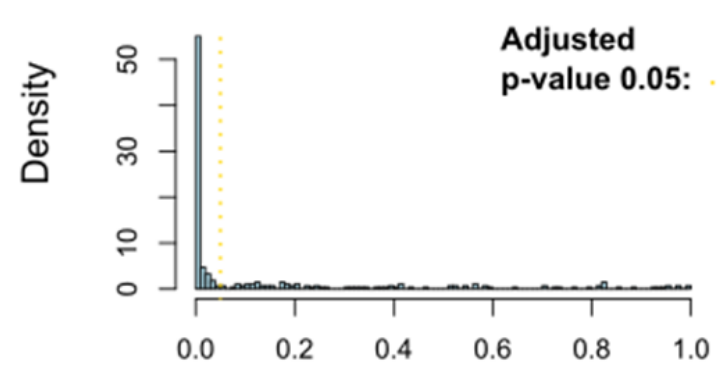

Correlation test p-value (adjusted)

C
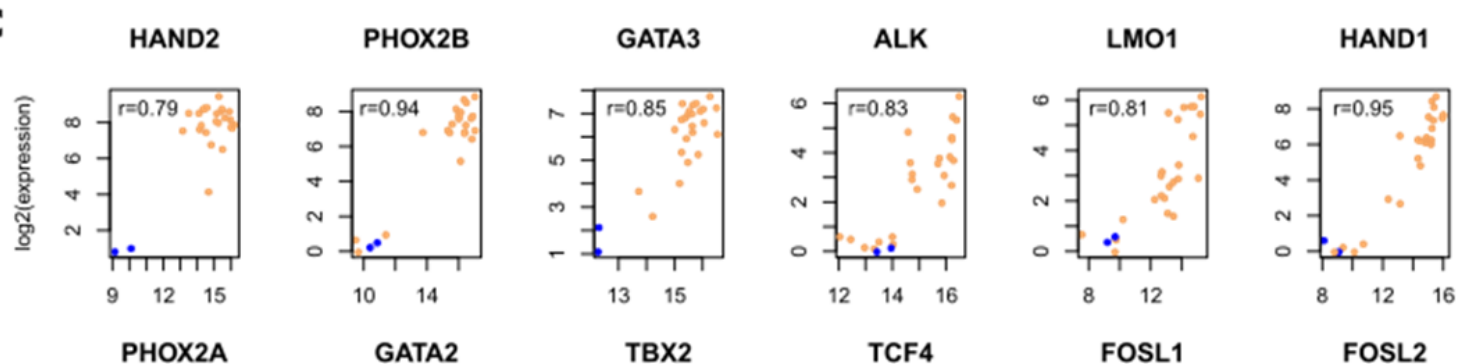

Samples:

PHOX2A
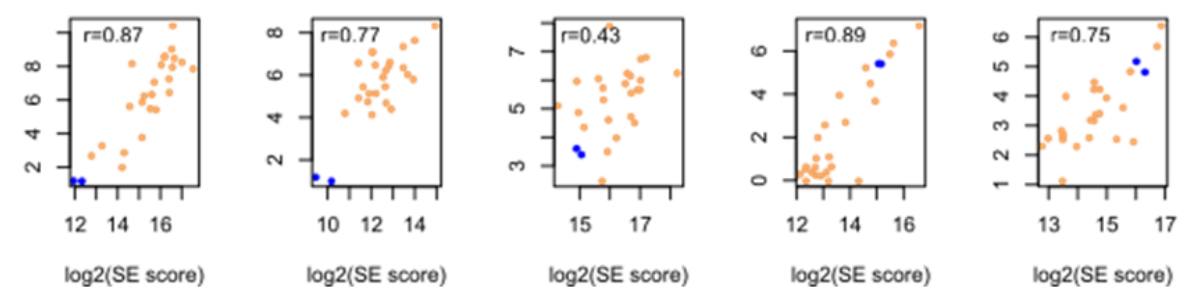

$\log 2$ (SE score)

$\log 2$ (SE score)

$\log 2$ (SE score)

910 Supplementary Figure 11: Gene expression linearly correlates with SE score (in log scale). a,

911 Pearson correlation coefficients were calculated for 267 SE regions corresponding to only one

912 gene and detected in at least 2 neuroblastoma samples. Pearson correlation one-sided 913 permutation tests were performed on the set of 25 neuroblastoma cell lines and $2 \mathrm{hNCC}$ samples;

914 p-values adjusted with the FDR method. $\mathbf{b}$, Distribution of corresponding adjusted p-values for 915267 SE regions. c, Examples of correlation between SE score and expression of particular genes. 916 Orange: neuroblastoma cell lines; blue: hNCC samples. 


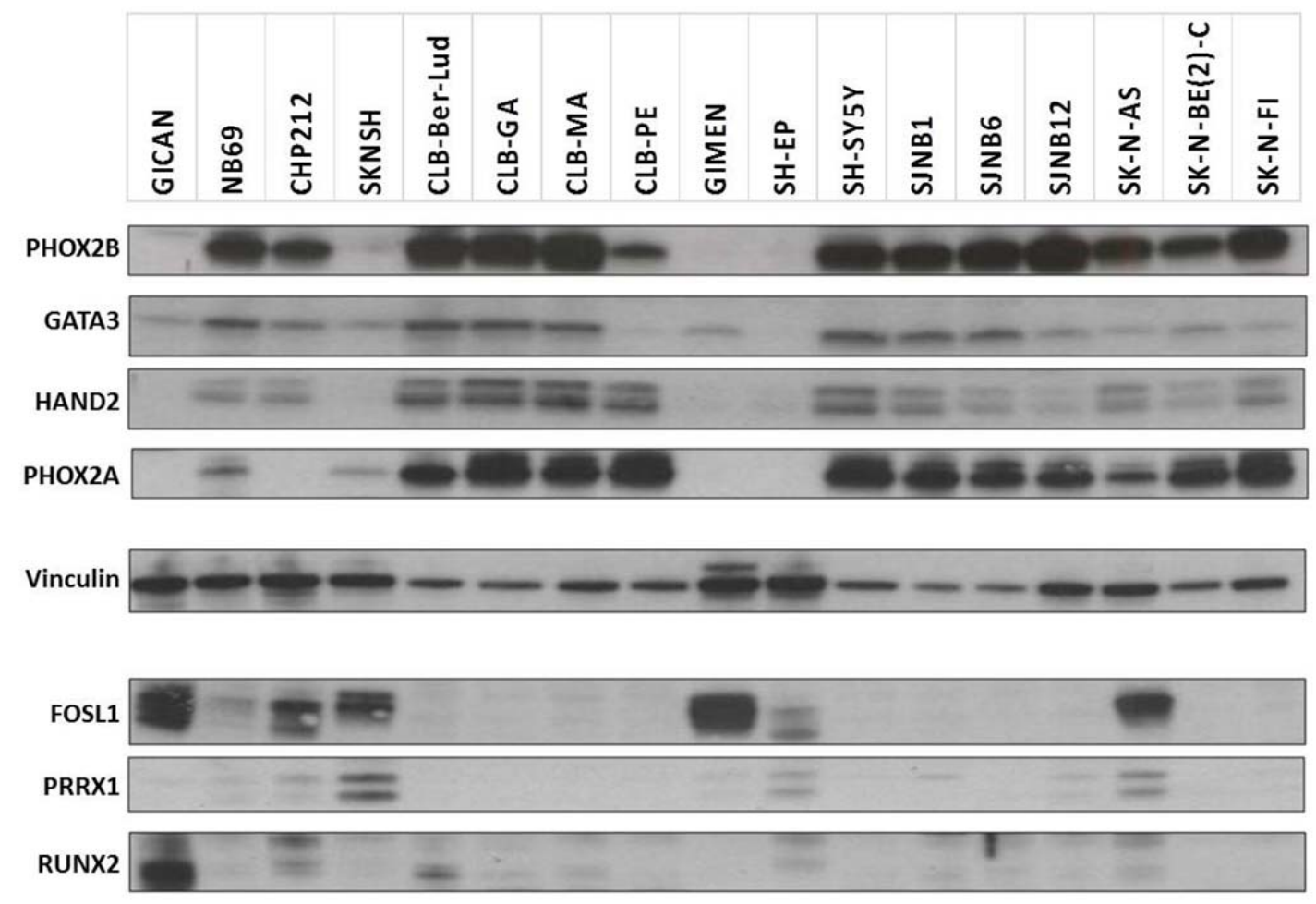

925 Supplementary Figure 12: Western blot analysis of PHOX2B, GATA3 (CST \#5852, D13C9), 926 HAND2 (sc-9409), PHOX2A (sc-8978), FOSL1 (sc-28310), PRRX1 (Sigma HPA051084), 927 RUNX2 (sc-101145) and vinculin in a panel of neuroblastoma cell lines. All antibodies were 928 used at 1:500 except the GATA3 antibody used at 1:1000. 


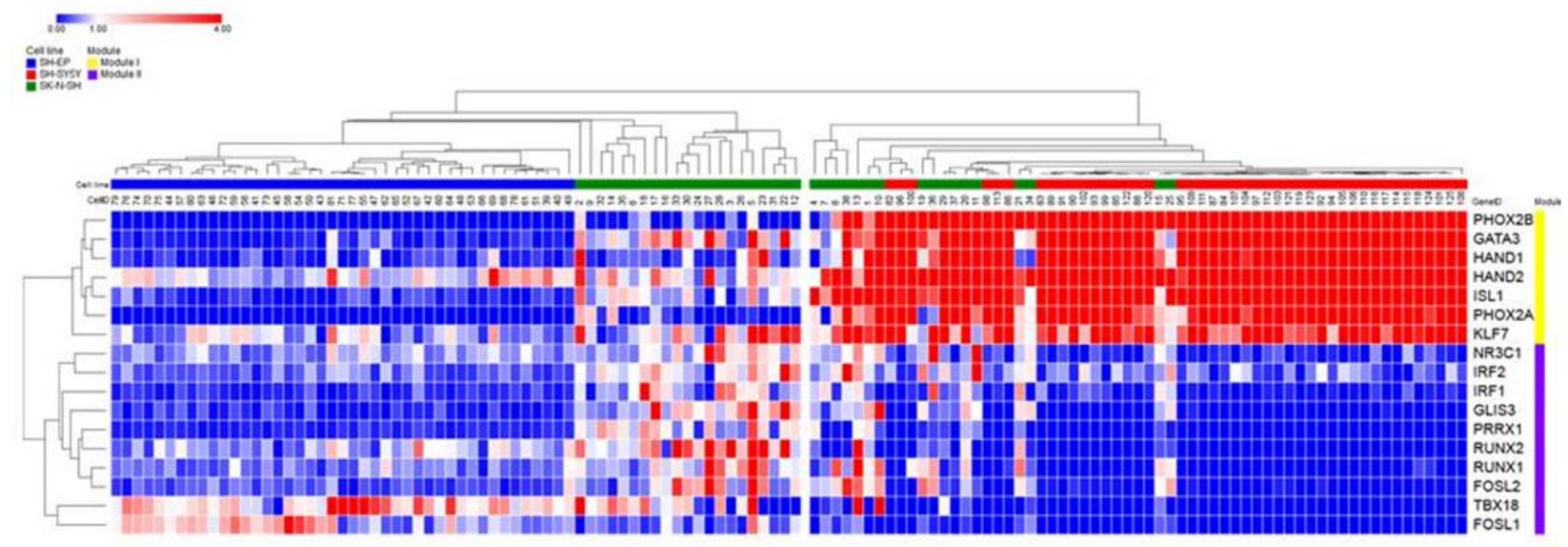

935 Supplementary Figure 13: Clustering of SK-N-SH, SH-EP and SH-SY-5Y single cells

936 analyzed by RT-q-PCR for the expression of TFs of modules 1 and 2. The first group of cells

937 includes all SH-EP cells as well as some cells of the SK-N-SH cell line; a second group includes

938 all SH-SY5Y cells as well as cells of the SK-N-SH cell line. RT-q-PCR data were normalized to

939 the SK-N-SH cell line population for the three cell lines using the geometric mean of the four 940 housekeeping genes. 


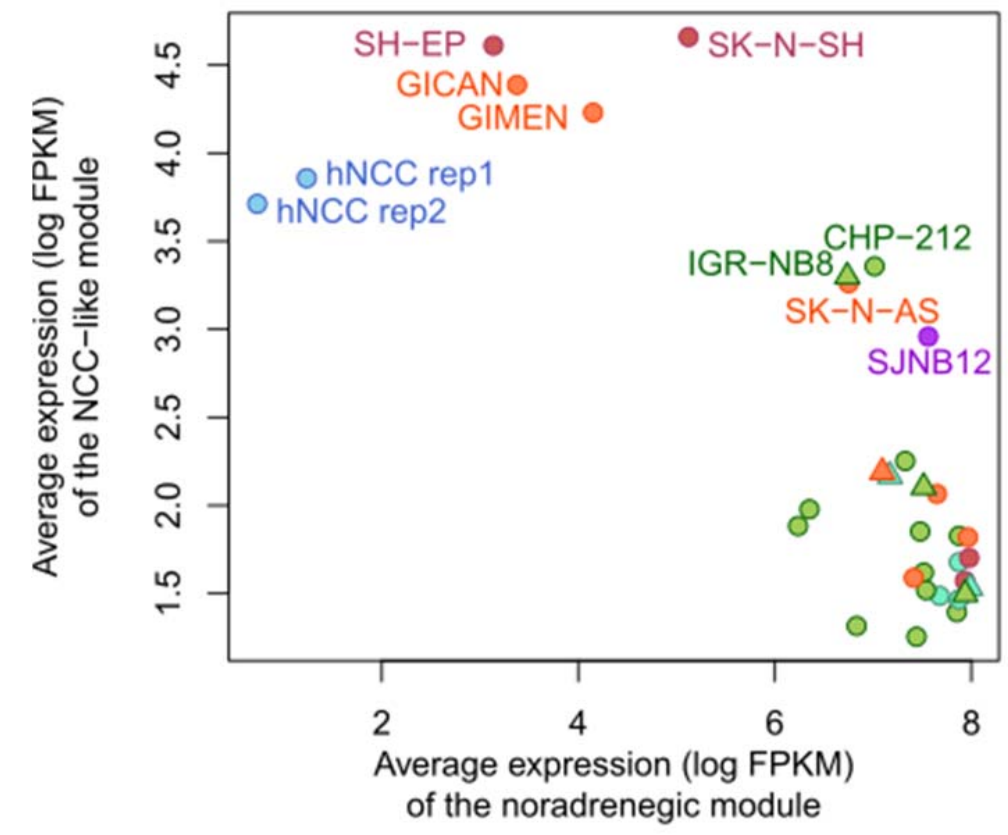

956 Supplementary Figure 14: Expression of modules 1 and 2 in neuroblastoma cell lines and 957 PDXs. Average is calculated for $\log 2$ FPKM values. 

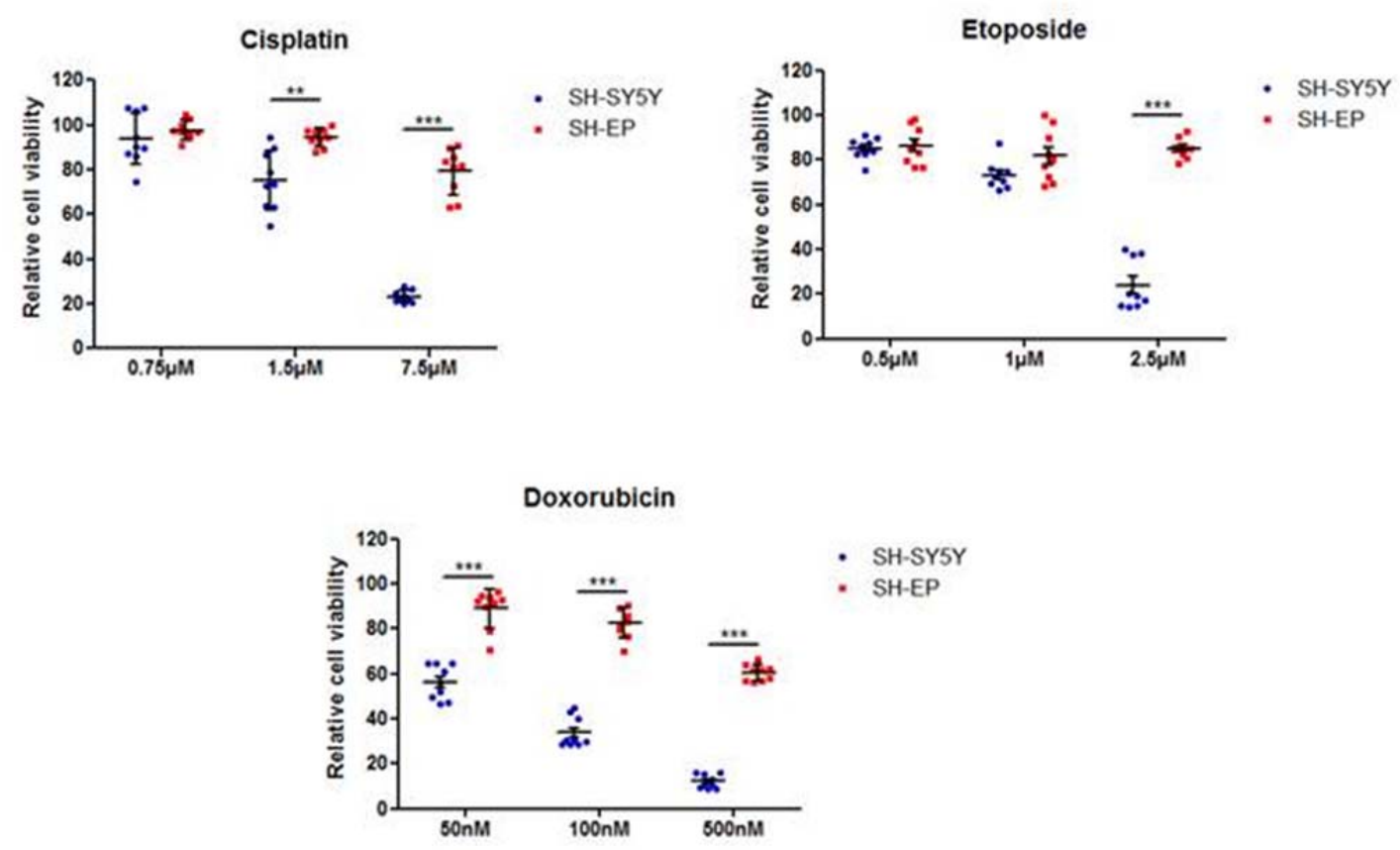

964

965

966 Supplementary Figure 15: NCC-like SH-EP cells are more resistant to chemotherapy than 967 noradrenergic SH-SY5Y cells ( $n=9$ technical replicates per condition; $P$ values were determined 968 via two-tailed unpaired Welch's t-test $(* * *: \mathrm{p}<0.001))$.

969

970

971

972

973 

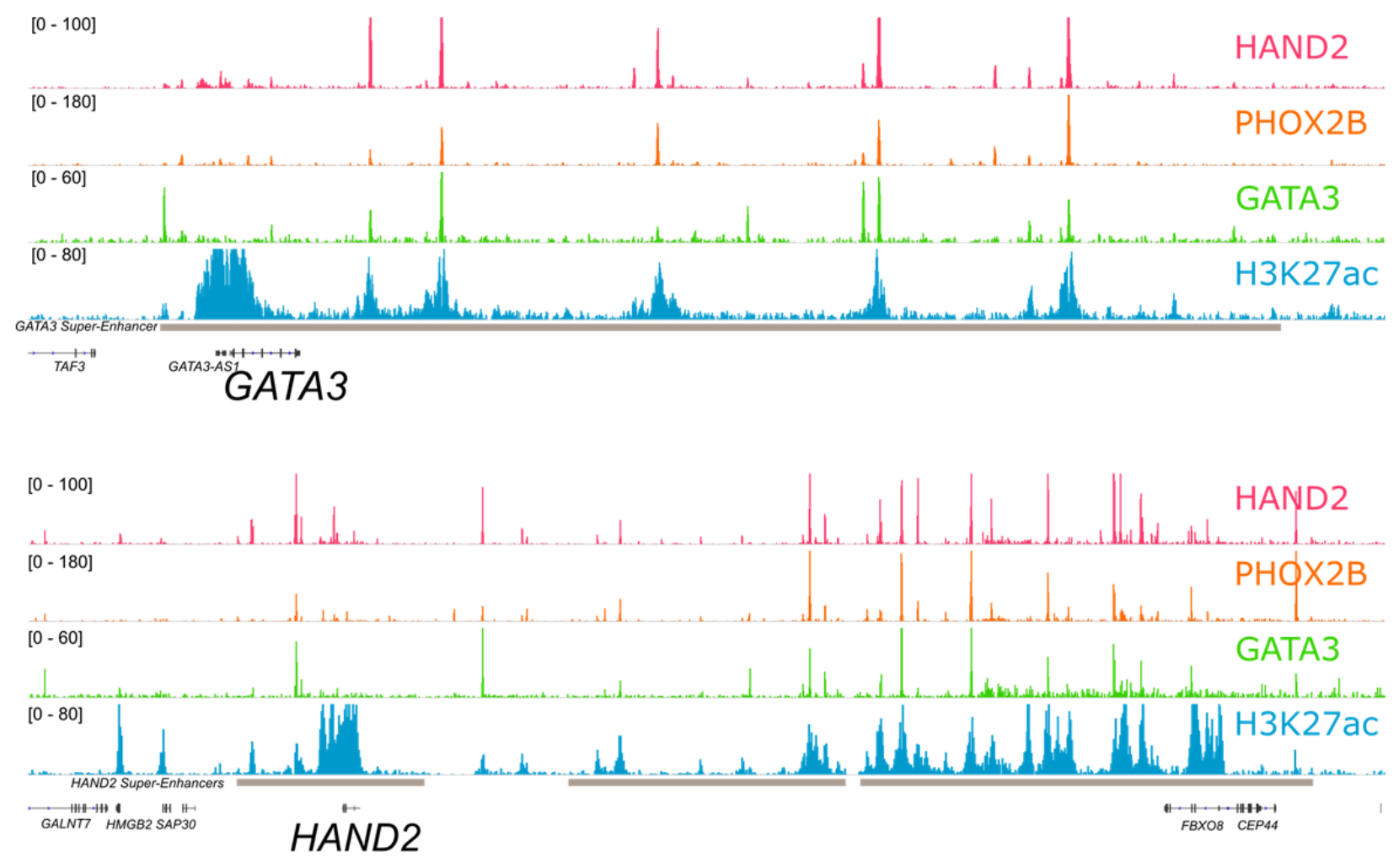

974
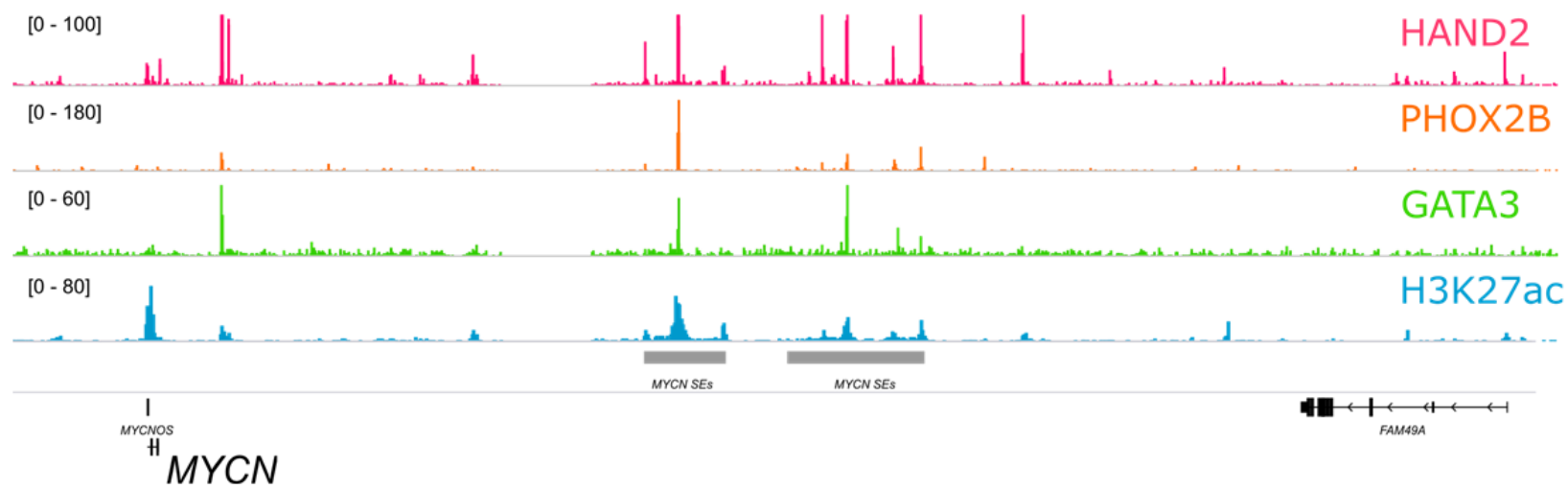

975

976

977

978 Supplementary Figure 16: Tracks for ChIP-seq profiles for PHOX2B, HAND2, GATA3 and 979 H3K27ac binding at the GATA3 (top), HAND2 (middle) and MYCN (bottom) SEs in the CLB980 GA cell line.

981

982 
a

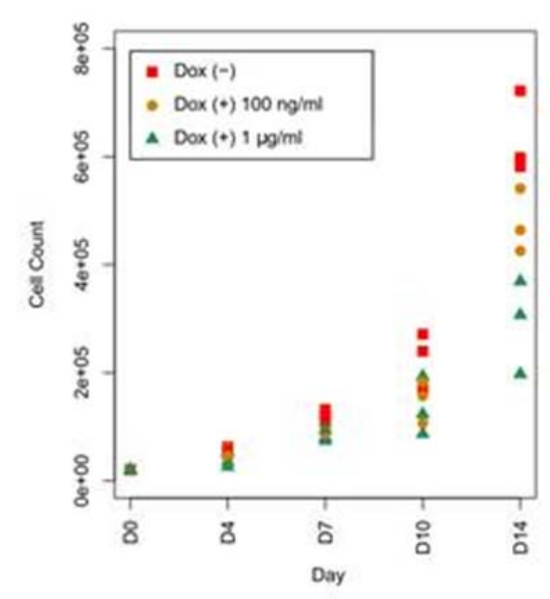

b

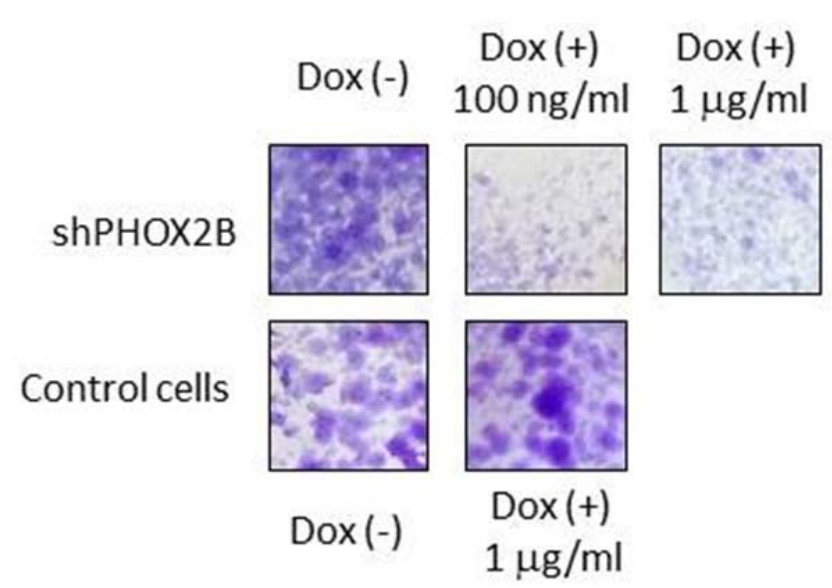

\section{C}

\section{sh1437}

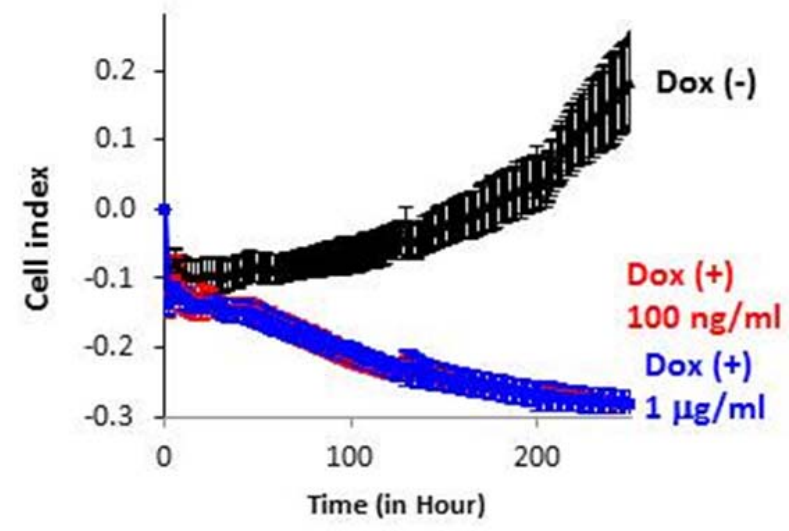

985 Supplementary Figure 17: a, Validation of $x$ CELLigence ${ }^{\mathrm{TM}}$ results by cell counting for the 986 CLB-GA cell line infected with the sh1783 vector targeting PHOX2B. $2 \times 10^{4}$ cells were plated in $98724-w e l l$ plates at day 0 in the absence or presence of doxycycline at $100 \mathrm{ng} / \mathrm{ml} \mathrm{or} 1 \mu \mathrm{g} / \mathrm{ml}$. The 988 number of living cells was counted at day 4, 7, 10 and 14. b, Decreased foci formation of CLB989 GA cells upon doxycycline-induced $P H O X 2 B$ knockdown. Doxycycline at $1 \mu \mathrm{g} / \mathrm{ml} \mathrm{did} \mathrm{not} \mathrm{affect}$ 990 growth of CLB-GA non-infected control cells. c, $x_{C E L L i g e n c e}{ }^{\mathrm{TM}}$ proliferation kinetics for the 991 SH-SY5Y cell line infected with the sh1437 vector targeting PHOX2B, respectively. Data shown 992 are the mean \pm s.d. of results obtained in the different conditions ( $n=5$ technical replicates) 993 
a
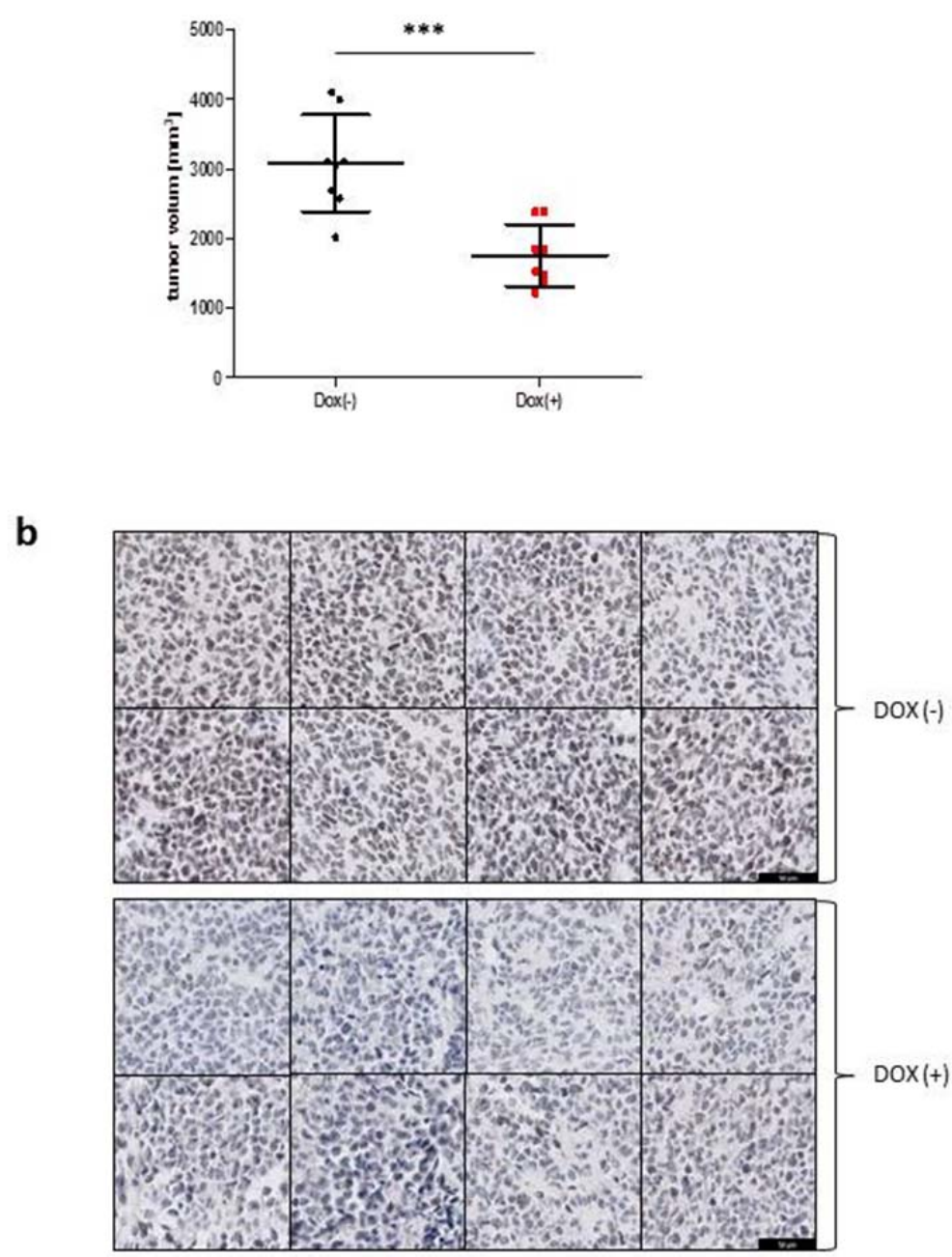

998 Supplementary Figure 18: a, Tumor volume of mouse xenografts of CLB-GA cells transduced 999 with sh1783 targeting PHOX2B after 11 days of treatment with sucrose alone (Dox(-)) or 1000 doxycycline and sucrose $(\operatorname{Dox}(+))$. b, PHOX2B expression analyzed by immunohistochemistry 1001 (EPR14423-Abcam) in mouse xenografts of CLB-GA cells transduced with sh1783 targeting 1002 PHOX2B treated or not with doxycycline (DOX). Each panel corresponds to a different tumor 1003 ( $\mathrm{n}=8$ tumors in each group).

1004 
a
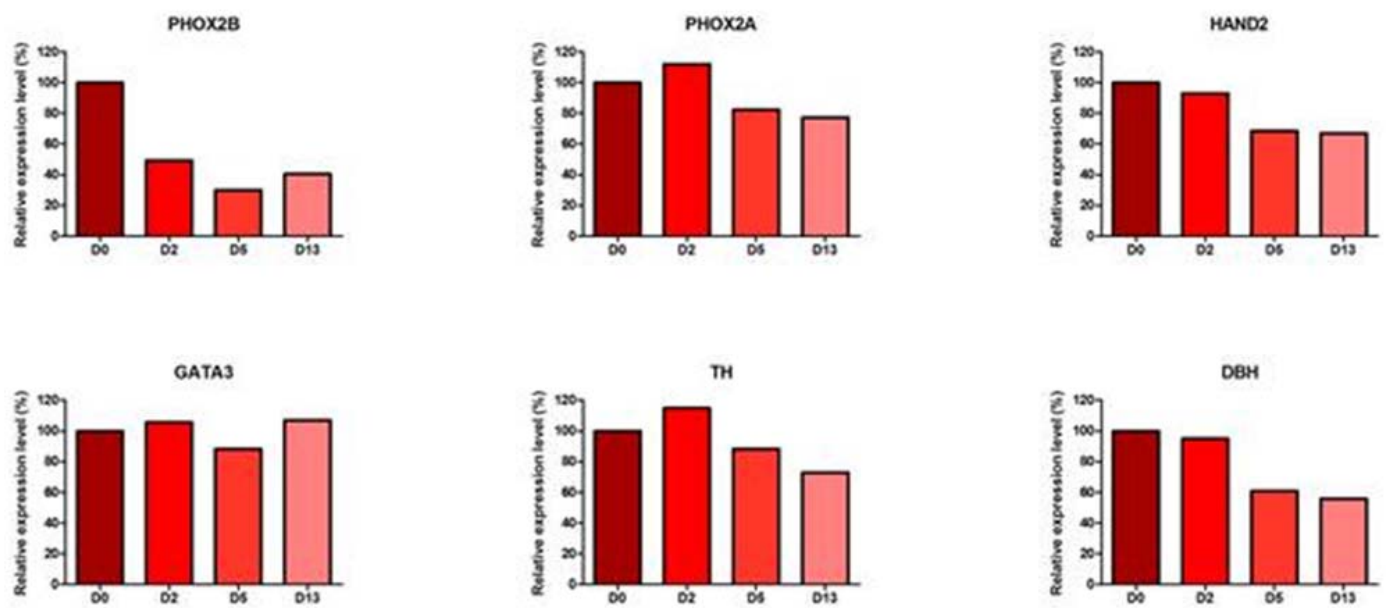

b
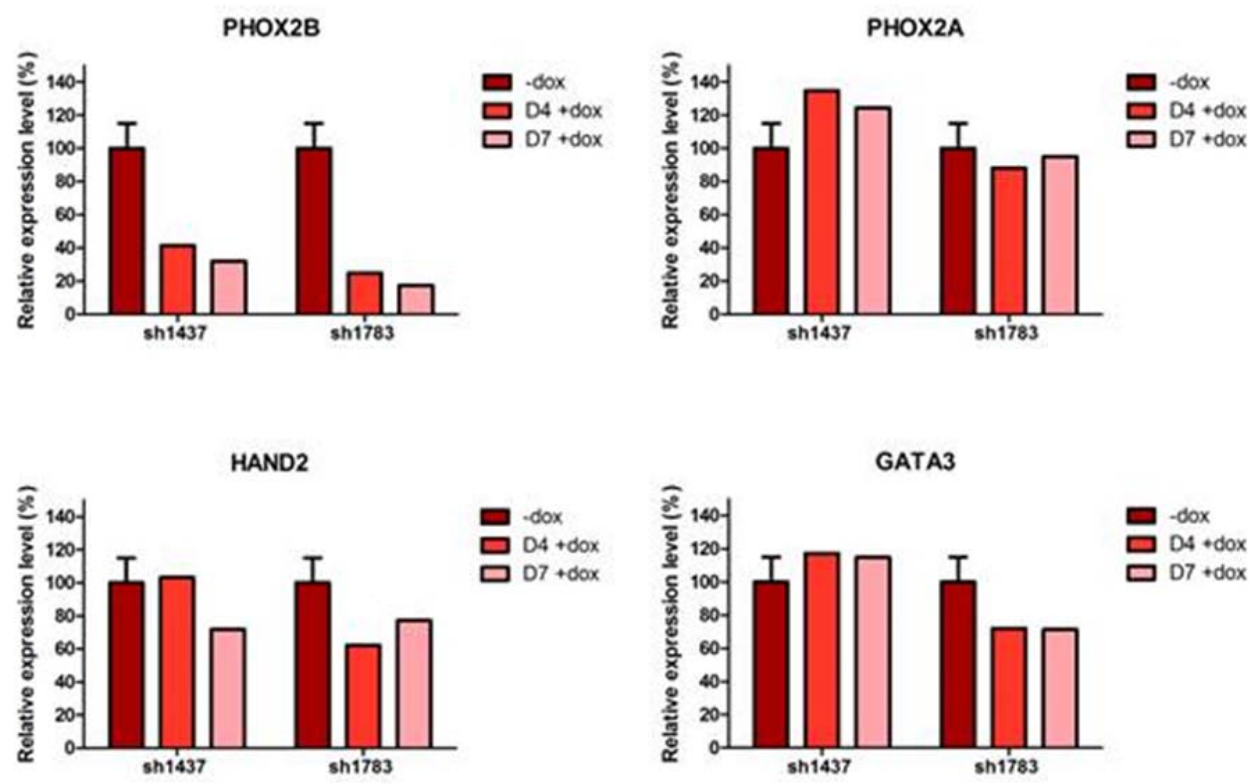

1006 Supplementary Figure 19: Impact of PHOX2B decrease on the expression profiles of CLB-GA 1007 and SH-SY5Y cells. a, RNA-seq was performed on CLB-GA cells transduced with sh1783 1008 targeting PHOX2B after 2, 5 and 13 days of doxycycline treatment and on untreated cells (D0). 1009 Expression levels (FPKM values) for each day are compared to the untreated cells (100\%). 1010 PHOX2B knockdown resulted in a modest decrease of PHOX2A, HAND2, TH and DBH. No 1011 expression of the genes of the NCC-like module was detected in any of the conditions. $\mathbf{b}$, 1012 Expression of genes of modules 1 and 2 was evaluated by RT-q-PCR on SH-SY5Y cells 
1013 transduced with sh1437 or sh1783 targeting PHOX2B after 4 and 7 days of doxycycline 1014 treatment and compared to untreated cells (100\%). GAPDH was used as a reference gene. No 1015 strong changes were observed following PHOX2B decrease. FOSL1, RUNX2 and PRRX1 were 1016 not detected neither in the untreated condition nor after PHOX2B knockdown. TaqMan(r) Gene 1017 Expression Assays (Thermo Fischer Scientific) used in this assays: GAPDH (4326317E), 1018 PHOX2B (Hs00243679_m1), HAND2 (Hs00232769_m1), GATA3 (Hs00231122_m1), 1019 PHOX2A (Hs00605931_mH), FOSL1 (Hs04187685_m1), RUNX2 (Hs01047973_m1), PRRX1 1020 (Hs00246567_m1).

1021

1022

1023

1024

1025

1026

1027

1028

1029

1030

1031

1032

1033

1034

1035

1036

1037

1038

1039

1040

1041

1042

1043 
a

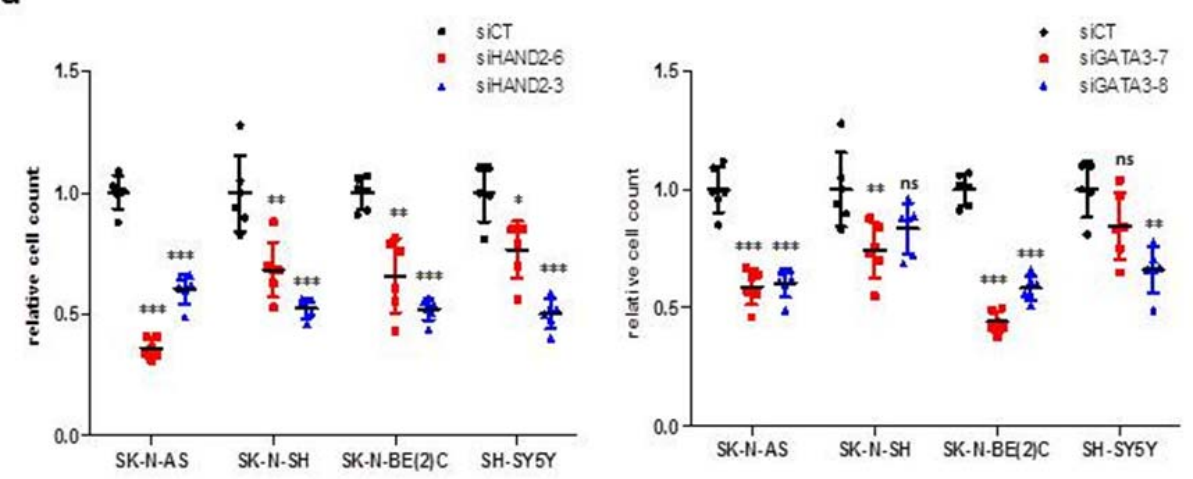

b

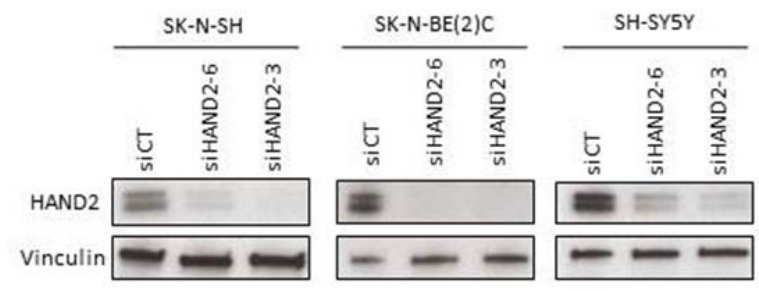

1045

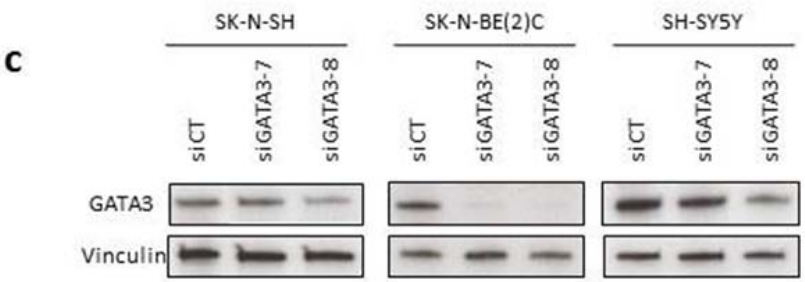

1046

1047

1048 Supplementary Figure 20: HAND2 and GATA3 knockdown impairs proliferation of SK-N-

1049 AS, SK-N-SH, SK-N-BE(2)C and SH-SY5Y cell lines. a, Cell counting of cells treated with

1050 siRNA targeting HAND2, GATA3 or with a control siRNA (at 3 days post-treatment for SK-N1051 BE(2)C, 5 days for SK-N-SH and 6 days for SK-N-AS and SH-SY5Y). (n=5 or 6 technical 1052 replicates, mean +/- s.d.). P values were determined via two-tailed unpaired Welch's t-test (***: $1053 \mathrm{p}<0.001)$. b and $\mathbf{c}$, Western blots for HAND2, GATA3, or vinculin. 
a

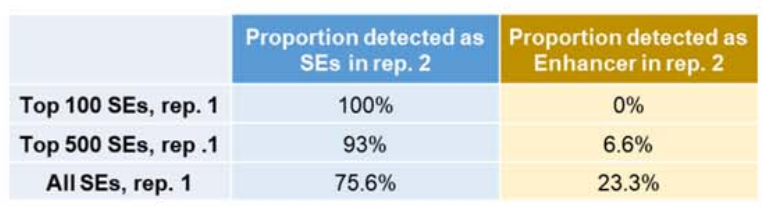

b

$\log ($ SE scores)

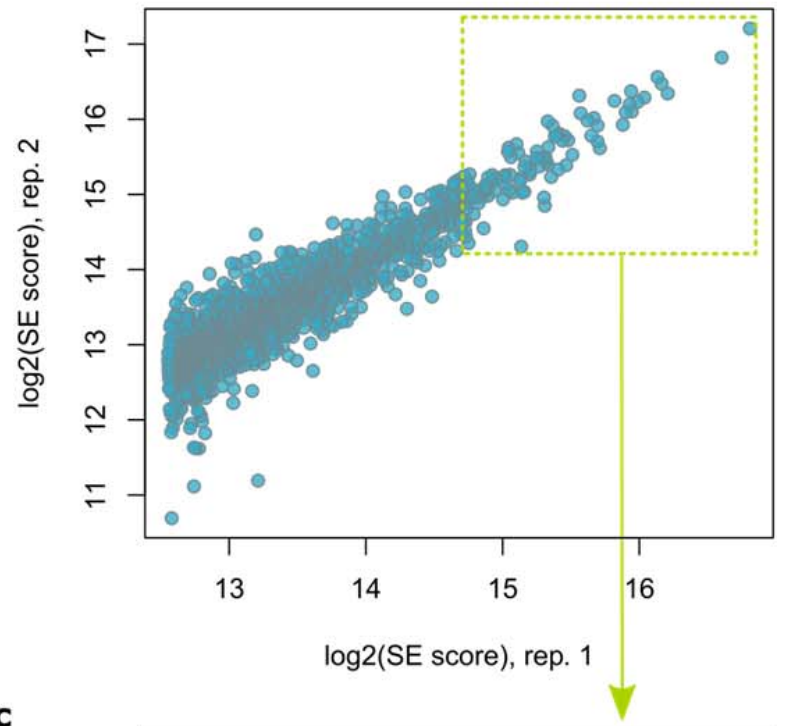

C

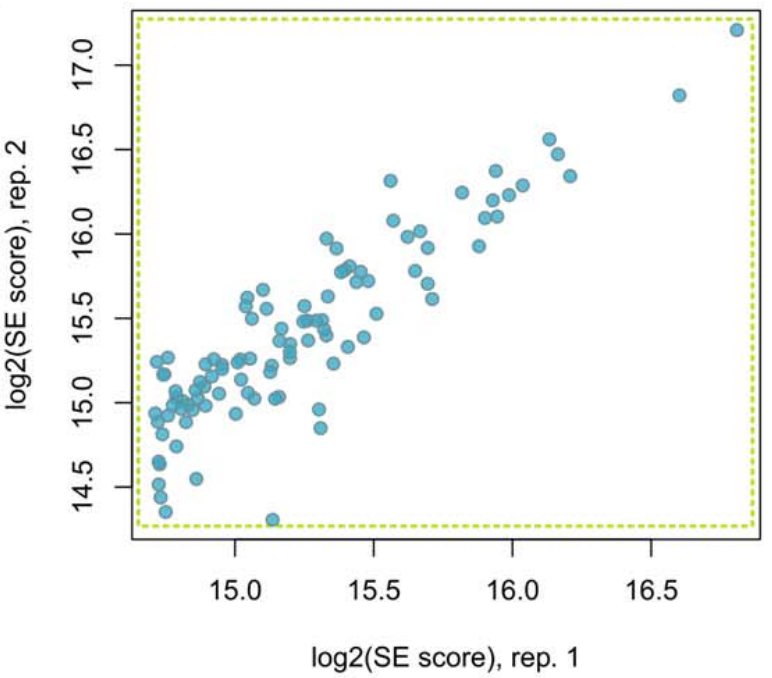

d

\begin{tabular}{|c|c|c|}
\hline & $\begin{array}{c}\text { Pearson correlation coef. } \\
\text { of SE log scores in rep. 1 } \\
\text { and rep.2 }\end{array}$ & $\begin{array}{c}\text { Spearman correlation } \\
\text { coef. of SE log scores in } \\
\text { rep. 1 and rep. 2 }\end{array}$ \\
\hline Top 100 SEs, rep. 1 & 0.90 & 0.86 \\
\hline Top 500 SEs, rep.1 & 0.92 & 0.88 \\
\hline All SEs, rep. 1 & 0.93 & 0.90 \\
\hline
\end{tabular}

1055 Supplementary Figure 21: Reproducibility analysis of SE calling and score assessment. ChIP1056 seq experiment for the H3K27ac mark was performed in duplicate for the CLB-GA cell line. a, 1057 Proportion of active SEs from replicate 1 detected as SEs in replicate 2. b, c, Correlation 
1058 between normalized values of SE scores in CLB-GA replicate 1 and 2, shown for all the SEs of 1059 replicate 1 (b) and the top 100 SEs of replicate 1 (c). d, Correlation coefficient for SEs scores 1060 between replicate 1 and 2 .

1061

1062

1063

1064

1065

1066

1067

1068

1069

1070

1071

1072

1073

1074

1075 


\section{Cluster Dendrogram}

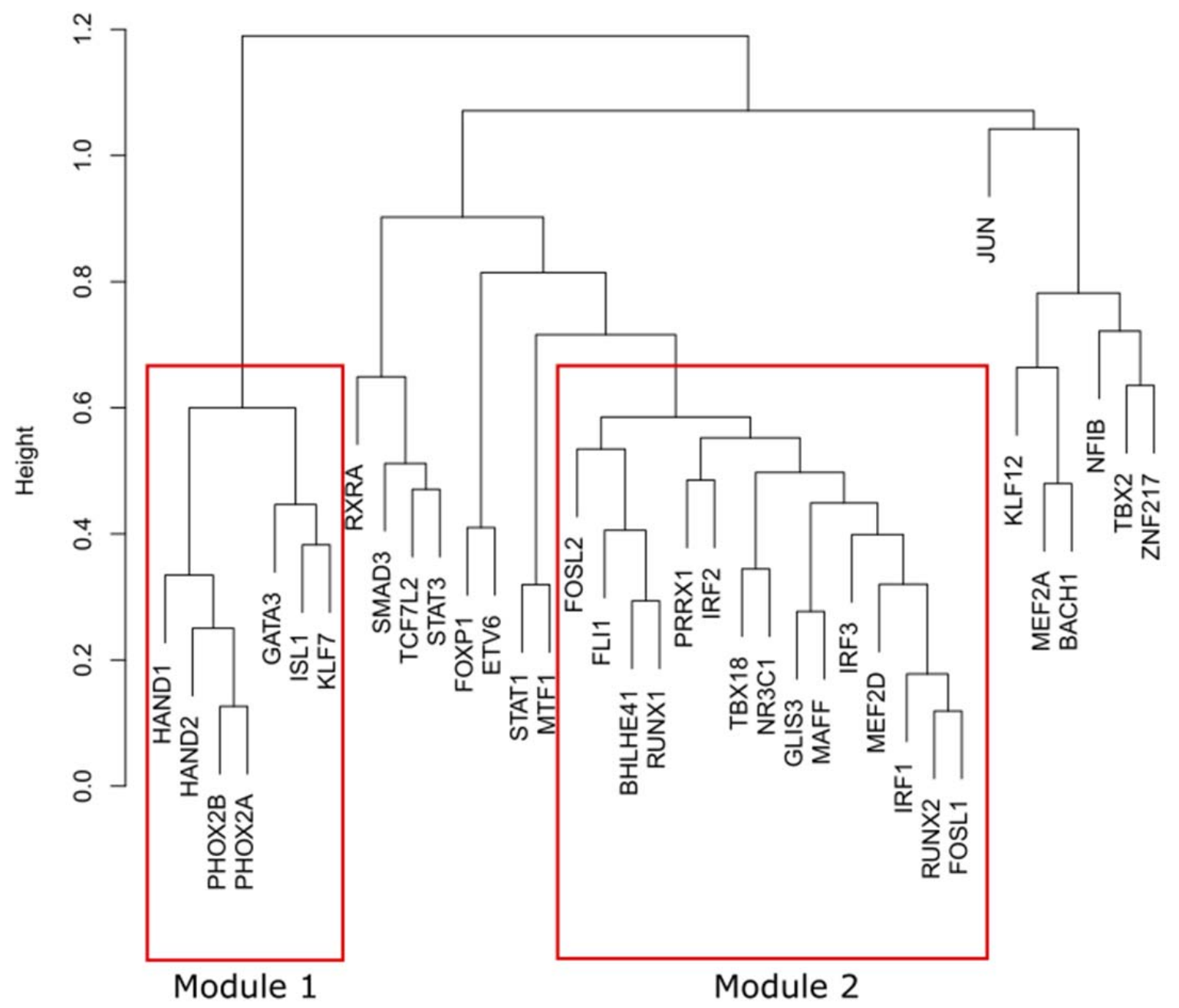

1076

1077 Supplementary Figure 22: Clustering of 37 genes from CRCs of neuroblastoma group I and II 1078 based on their expression correlation in NB cell lines and PDX (R package 'hclust' with the 1079 McQuitty method). Two modules were defined. 